\title{
„Wir wollen Werte schaffen für die Gesellschaft" - Der Public Value im Spannungsfeld zwischen Aktienwert und Gemeinwohl. Eine Fallstudie am Beispiel der Deutsche Börse AG
}

\author{
Börsen; Gemeinwohl; Management; Public Value
}

Als börsennotierte Aktiengesellschaft, die mit der Frankfurter Wertpapierbörse und der Eurex Deutschland teilrechtsfähige Anstalten des öffentlichen Rechts betreibt, ist die Deutsche Börse AG mehr als „nur" ein gewinnorientiertes Unternehmen. Aufgrund ihrer besonderen Rechtsstruktur muss sie ihr Handeln sowohl aus privatwirtschaftlicher als auch aus gesellschaftspolitischer Perspektive rechtfertigen. Was sind konkrete Wertbeiträge, welche Manager der Deutschen Börse in diesem Kontext als Gemeinwohlbeiträge (Public Value) ihrer Unternehmung identifizieren? Und in welchem Verhältnis stehen diese Beiträge zueinander? Die vorliegende Untersuchung beantwortet diese Fragen im Rahmen einer qualitativen Fallstudie. Im Ergebnis zeigt sich, dass vor allem die funktionale Aufgabenerfüllung als gesellschaftlich wertvoll gesehen wird. Der vor dem empirischen Hintergrund erarbeitete Bezugsrahmen ermöglicht es, hierbei zwischen Wertbeiträgen als konkurrierenden und ergänzenden Unternehmenszielen zu unterscheiden. Daraus folgende Implikationen für Theorie und Praxis werden diskutiert.

\section{Einleitung}

Börsenorganisationen haben eine hervorgehobene Bedeutung für das politische und ökonomische Funktionieren marktwirtschaftlich organisierter Volkswirtschaften. „Die Börse ist eine Einrichtung des modernen Großhandelsverkehrs. Ihre Unentbehrlichkeit für die moderne Wirtschaftsweise beruht auf dem gleichen Grunde, aus welchem die moderne Form des Handelsverkehrs überhaupt erwachsen ist", hielt bereits Max Weber im Jahr 1894 fest (1999 [1894] S. 135). Andere Autoren wie z. B. George Gibson (1891) formulieren zu jener Zeit sogar noch plakativer, so in einem Ratgeber für Investoren von 1857: „The stock exchange is more powerful than the monarch “ (in: Preda 2009, S. 188). Oder: „The stock exchange may... fulfill specific economic functions, but beyond that, it is a spiritual force in itself and, as such, it brings the nation together: this is its true ethical quality“ (zit. nach Preda 2009, S. 189).

Die Gedanken an eine ökonomische „Unentbehrlichkeit“ und gesellschaftliche Relevanz von Börsen sind nach wie vor aktuell. Auf Finanz- und Kapitalmärkten finanzieren Unternehmen und öffentliche Haushalte ihre Aktivitäten, investieren Bürger in ihre Altersvorsorge oder sichern sich verschiedene Akteure - von der internationalen Unternehmung bis hin zum lokal produzierenden Getreideerzeuger - gegen so unterschiedliche Risiken wie Wechselkursschwankungen und Ern- 
teausfälle ab. Finanzmärkte haben damit nicht nur wirtschaftliche, sondern auch politische und gesellschaftliche Funktionen.

Gleiches gilt für die Organisationen, die diese Märkte betreiben. Denn indem sie die technologischen und häufig auch regulatorischen Rahmenbedingungen mitgestalten, die die oben genannten Aktivitäten ermöglichen, kommt diesen Organisationen mehr als „,nur“ eine ökonomische Funktion zu. Dementsprechend stellen sich Fragen nicht nur zu wirtschaftlichen sondern auch zu gesellschaftspolitischen Implikationen, welche mit den unterschiedlichen Organisationsformen und dem Management von Börsenorganisationen einhergehen. Diese Fragestellungen sind auch deshalb interessant, da die Organisation öffentlicher Finanz- und Kapitalmärkte durchaus unterschiedliche Formen annehmen können. Dies belegen u.a. historische Erfahrungen der drei großen Börsen des ausgehenden 18. und 19. Jahrhunderts. Demnach kann ein regulierter Kapitalmarkt sowohl durch von der Regierung geschaffene Akteure des öffentlichen Rechts (Paris) als auch durch private Korporationen als Unternehmung (London) oder als „Club“ (New York) betrieben werden (Davis/Neal 1998, S. 40).

Über diese historische Dimension hinaus sind Organisations- und Regulierungsfragen von Kapitalmärkten von unmittelbarer Relevanz für aktuelle Gestaltungsaufgaben in Politik und Wirtschaft. Im Zuge der globalen Finanz- und Wirtschaftskrise haben Regierungen rund um den Globus Finanzorganisationen durch Rettungsmaßnahmen und Garantien unterstützt (vgl. Fischer/ Hainz/Rocholl/Steffen 2012, S. 2). Vornehmliche Begründung hierbei war der Hinweis auf die systemische Relevanz besagter Organisationen, deren Liquidierung zu Schieflagen im gesamtwirtschaftlichen Gleichgewicht geführt hätte (ibid.). Börsenorganisationen sind keine Banken. Ähnlich wie Banken stellen sie jedoch den Teilnehmern einer Volkswirtschaft Infrastrukturen zur Kapitalaufnahme zur Verfügung und wirken so im Allokationsprozess von finanziellen Ressourcen mit. Die Qualität dieser Infrastrukturleistungen hat somit weithin wahrnehmbare Folgen. Dies illustrieren Störungen im börslichen Allokationsprozess, wie z. B. der „Flash Crash“ in den USA im Mai 2010, in dessen Folge der Dow Jones rund 1 Billion USD an Wert verlor (Sornette/von der Becke 2011).

Börsen gehören also wie Banken zu den systemrelevanten Finanzinfrastrukturen einer Marktwirtschaft. Ebenso wie Banken tragen Börsen zur Kapitalversorgung einer Volkswirtschaft bei. Ihnen kommt durchaus der Charakter von Infrastrukturanbietern zu. Ähnlich einem Telekommunikationsdienstleister in der Realwirtschaft ermöglichen sie den Austausch von Informationen in Form von Kurspreisen und Indizes. In Analogie zu einem als Warenumschlagplatz fungierenden See- oder Binnenhafen operieren sie zudem als Plattform für den Handel mit Finanzwerten. Sie sind somit Dreh- und Angelpunkt moderner kapitalistischer Wirtschaften.

Als börsennotiertes Unternehmen, das mit der Frankfurter Wertpapierbörse (FWB) und der Eurex Deutschland teilrechtsfähige Anstalten des öffentlichen Rechts betreibt, vereint die Gruppe Deutsche Börse (GDB) mehrere der benannten Traditionen und Funktionen innerhalb ihres organisatorischen Profils. Sie selbst besitzt nicht die Rechtspersönlichkeit eines öffentlich-rechtlichen Akteurs, ist jedoch auch mehr als ein gewinnorientiertes Unternehmen. Aufgrund dieser besonderen Ausgangslage muss die Deutsche Börse ihr Handeln somit sowohl in privatwirtschaftlicher Sicht gegenüber ihren Aktionären als auch aus öffentlich-rechtlicher und gesellschaftspolitischer Perspektive rechtfertigen. Dodd Jr. reflektierte bekanntlich die gesellschaftspolitischen Legitimationen von Unternehmungen, indem er darauf hinwies, dass ihr „,business is ... encouraged by 
the law primarily because it is of service to the community rather than because it is a source of profits to its owners“ (1932, S. 1149). Eine damit verbundene gesellschaftliche ,licence to operate" kann schwerlich allein anhand von Marktinformationen beschrieben werden. Eine Steigerung von Marktgrößen wie Umsatz, Profit und Aktienwert der Deutsche Börse AG (DBAG) reicht folglich nicht aus, um Existenz und Handeln der Gruppe Deutsche Börse und ihrer Angestellten in gesellschaftlich-politischen Kontexten zu legitimieren. Erst empirisch abgestützte Aussagen zum wahrgenommenen gesellschaftlichen Wertbeitrag ermöglichen eine Bewertung in diesem Bereich.

Wir möchten in dieser Studie einen Beitrag zur Charakterisierung der unterschiedlichen Zieldimensionen unternehmerischen Handelns leisten. Im Spannungsfeld von Wirtschaft, Staat und Gesellschaft bietet sich eine Untersuchung der Fragestellung bei der Gruppe Deutsche Börse an, weil sie als privatwirtschaftliche und ihren Aktionären verpflichtete Unternehmung stets auch einen Auftrag im Rahmen der Daseinsvorsorge moderner marktwirtschaftlich organisierter Gesellschaften hat. Der Organisationszweck der Deutschen Börse legt allein der Form nach divergierende Partikular- und Gemeinwohlinteressen nahe. Einerseits ist sie den Aktionären verpflichtet, andererseits nimmt sie eine öffentliche, dem Gemeinwohl verpflichtete Funktion war. Welches sind in diesem Kontext die von der Börse geleisteten und zu leistenden Wertbeiträge zum Gemeinwohl, die ihre gesellschaftliche „licence to operate“ bzw. ihren „Public Value“ kennzeichnen (Meynhardt 2008; 2009)? Wo liegen wahrgenommene Konfliktfelder?

Wir nähern uns diesen Fragen aus einer Managementperspektive und untersuchen, wie Manager aus organisationsinterner Sicht den Wertbeitrag der Deutschen Börse (re)konstruieren. Auf der Managementebene treffen unterschiedliche sachliche, juristische oder auch politische Spannungsfelder aufeinander und müssen in strategische und operative Überlegungen einbezogen werden. Zur Erfassung der Erfahrungswelt von Managern wählen wir ein qualitatives Fallstudiendesign, um diese Spannungsfelder und die diesbezüglichen Sichtweisen der Befragten auf geleistete Wertbeiträge dem Gegenstand angemessen abbilden zu können (Eisenhardt/Graebner 2007; Siggelkow 2007).

Zunächst zeigen wir Forschungslücken zum Management von privatwirtschaftlichen Unternehmen mit einer öffentlich-rechtlichen (Teil-) Funktion auf und stellen kurz den „Public Value“Ansatz als konzeptionellen Rahmen unserer Studie vor. Nach der Betrachtung des Forschungskontextes beschreiben wir den methodischen Ansatz und das Vorgehen der Datenanalyse. In einzelnen Schritten werden dann die Ergebnisse vorgestellt und diskutiert. Die Studie schließt mit den Limitationen und einem Ausblick auf Möglichkeiten weiterer Forschung.

\section{Literaturanalyse}

In Folge der von Rupert Windisch (1987) herausgegebenen Erörterungen zur Privatisierung staatlicher Monopole in Deutschland (z. B. Deutsche Lufthansa oder Deutsche Telekom) wurden Fragen der Erfüllung aktuell oder ehemals hoheitlich definierter Aufgaben durch Unternehmen unter verschiedenen Blickwinkeln diskutiert. Ein Beispiel dafür sind die Analysen zu Maßnahmen des Veränderungsmanagements bei der Deutsche Lufthansa AG nach ihrer Privatisierung (Bruch/ Sattelberger 2001 a; b). Die dabei untersuchten organisationalen Wandelprozesse bei der Über- 
führung von Staatsunternehmen in private Eigentümerstrukturen geben indirekt Aufschluss über neue Managementherausforderungen in Organisationen, die sich dem Ziel der Gewinnmaximierung verpflichten, aber weiterhin einen öffentlich-rechtlichen Auftrag erfüllen. Ein direkter $\mathrm{Zu}-$ gang zu Erörterungen managerieller Sichtweisen in Bezug auf Handeln und Legitimation von Unternehmen mit besonderer gesellschaftlicher Relevanz bietet sich über die Forschung zum Management öffentlich-privater Partnerschaften (ÖPP) an. Denn gerade im ÖPP-Bereich gilt, dass Unternehmen zwar öffentlich-rechtlich determinierte Leistungen erbringen, ihre Manager jedoch Anreiz- und Entscheidungsmechanismen unterliegen, welche sich von Steuerungsmechanismen innerhalb des öffentlichen Sektors unterscheiden. Der von uns postulierten Frage, wie Manager mit daraus erwachsenden ambivalenten Herausforderungen umgehen, ist bisher wenig Aufmerksamkeit geschenkt worden. Weihe formuliert diesen Befund deutlich mit dem Hinweis, dass im Bereich des ÖPP-Managements "operational practice has been more or less black-boxed" (2008, S. 153).

In allgemeiner Weise berührt unser Forschungsinteresse auch aktuelle Überlegungen zum Management von Finanzorganisationen nach der Finanzkrise. Hierzu gehören sowohl Corporate Governance-Fragen in Bezug auf Finanzinstitutionen (Pirson/Turnbull 2011) als auch und vor allen Dingen Fragen des Stakeholder-Managements von Finanzinstitutionen (O’Sullivan/O'Dwyer 2009; Freixas 2010; Schanz 2011; Werhane/Hartman/Archer/Bevan/Clark 2011).

Einen unmittelbaren Ansatzpunkt für den deutschen Kontext bietet die Studie „Der öffentliche Auftrag der deutschen Sparkassen aus der Perspektive des Stakeholder-Managements“ (Brämer/ Gischer/Pfingsten/Richter 2010). Die Autoren kommen dort zu dem Schluss, dass der von den Sparkassen in Deutschland zur Legitimation ihrer Existenz und ihres Handelns verwendete Begriff des öffentlichen Auftrags ,einem zeitlichen Wandel unterliegt und mitnichten statischer Natur ist“ (ibid. S. 329). Hierbei ,stellen sie fest, dass der öffentliche Auftrag von den Sparkassen selber teilweise anders ausgelegt und kommuniziert wird, als er aus rechtlicher und aus wissenschaftlicher Sicht zu verstehen ist" (Summermatter 2012, S. 79).

Edeling (2002) bemerkt, dass sich die „Einflüsse von Marktöffnung und Wettbewerbsorientierung“ im Bereich kommunaler Versorgungsunternehmen auf die Zielorientierung von deren Managern dahingehend ausgewirkt haben, ,dass das öffentliche Interesse zunehmend auf rein fiskalische Ziele beschränkt wird,... [womit schlussendlich], der kommunalen Wirtschaftstätigkeit ihre Legitimationsgrundlage'... entzogen wird“" (zitiert nach: Summermatter 2012, S. 78 f.). Wie Brämer et al. (2010) aufzeigen, neigen Finanzorganisationen wie Sparkassen dazu, ihren öffentlichen Auftrag selbst aktiv zu definieren. Edeling (2002) folgend könnte man vermuten, dass eine stärkere Einbindung von Finanzorganisationen in den globalen Wettbewerb dazu führt, dass die Manager besagter Organisationen ihre Zielorientierungen stärker zugunsten betriebswirtschaftlicher Faktoren und Finanzgrößen ausrichten. Andere Legitimationsmuster organisationalen Handelns müssten demnach fortan hinten anstehen. Andererseits ließe sich argumentieren, dass die regulatorischen und politischen Folgen der Finanz- und Wirtschaftskrise dazu führen, dass die Manager von Finanzorganisationen Gemeinwohlfragen mehr Beachtung schenken.

Zusammenfassend ist erkennbar, dass die Fragen nach der Ausrichtung von manageriellen Zielorientierungen in jenen Finanzorganisationen, welche aufgrund ihrer rechtlichen Verfasstheit sowohl den Gedanken des Gemeinwohls als auch des „Shareholder Value“ verpflichtet sind, kaum empirisch erforscht sind. Die hier wegweisende Arbeit von Brämer et al. (2010) konzentriert sich 
allein auf den Gemeinwohlauftrag von Sparkassen als Anstalten des öffentlichen Rechts. Tse (2011) schätzt ein, dass die unbefriedigende theoretische Durchdringung der Rolle unterschiedlicher Stakeholder in Bezug auf das Management finanzwirtschaftlicher Organisationen u. a. auch durch das Fehlen praktischer Lösungen erklärt wird, denn ,the tools and mechanisms needed to put the stakeholder theoretical framework into practice are yet to be developed" (S. 59 f.).

Den oben festgestellten Lücken in der Literatur entsprechend fokussiert der vorliegende Beitrag die Frage, inwieweit leitende Angestellte der GDB Widersprüche und Spannungsfelder innerhalb ihres unternehmerischen Gestaltungsauftrags identifizieren. Handlungsleitend ist dabei für uns der Public Value-Ansatz, in dem unterschiedliche Bewertungsdimensionen erfasst werden.

\section{Public Value als konzeptioneller Rahmen}

Wie kann man Wertbeiträge jenseits eines ökonomischen „value added“ erfassen? Bei der Beantwortung dieser Frage sind Sichtweisen denkbar, nach welchen der Legitimation stiftende ,Sozialvertrag“ (vgl. Donaldson/Dunfee 1999) auf der Bewahrung und Durchsetzung ökonomischer, rechtlicher aber auch ethischer Institutionen beruht (z. B. Knight 1982 [1939]; Donaldson/Dunfee 1999; Schumpeter 2008 [1934]). Da jedoch nicht alle diese Leistungen und Funktionen auf Märkten gehandelt und mit einem Preis versehen werden, können Märkte auch nicht als alleinige Informationsquellen zur Beantwortung dieser Frage dienen. Ein alternativer Analyseansatz ist notwendig, der ergänzende Bewertungskriterien bereitstellt.

Konzeptioneller Ausgangspunkt für unsere Fragestellung ist die Managementperspektive. In Ergänzung zu normativen Ansätzen, welche rechtliche oder ethische Verpflichtungen organisationalen Handelns diskutieren, steht für uns eine positivistische Sichtweise auf die gesellschaftliche Wertschöpfung der GDB im Mittelpunkt. Wir untersuchen nicht, welche gesellschaftlichen Wertbeiträge der Gesetzgeber von der GDB erwartet oder welche normativen Leitlinien das Handeln von deren Managern aus wirtschaftsethischer Perspektive bestimmen sollten. Mit dem Begriff des „Public Value“ (Meynhardt 2008; 2009) wird vielmehr ein konzeptioneller Rahmen gewählt, welcher bestehende gesellschaftliche Verpflichtungen der GDB aus der Sichtweise der Befragten rekonstruiert.

Gesellschaftliche Werte, im Sinne eines „Public Value“ werden „geschaffen oder zerstört, wenn das individuelle Erleben und Verhalten von Personen und Gruppen so beeinflusst wird, dass dies stabilisierend oder destabilisierend auf Bewertungen des gesellschaftlichen Zusammenhalts, das Gemeinschaftserleben und die Selbstbestimmung des Einzelnen im gesellschaftlichen Umfeld wirkt" (Meynhardt 2008, S. 462). Mit dieser Begriffsbestimmung werden einerseits Gemeinwohltheorien in einen Wertschöpfungskontext gestellt, der über die Managementebene nach Zugängen sucht, um tatsächlich geleistete Beiträge (,Wertbeiträge“) von Organisationen jenseits von Märkten als Bezugsrahmen zu analysieren. Andererseits erfolgt darüber eine enge Verknüpfung des Gemeinwohlbegriffs mit psychologischen Theorien menschlicher Bedürfnisse und Werte. Von gesellschaftlicher Wertschöpfung wird erst gesprochen, wenn damit eine Wahrnehmung auf der Individualebene verbunden ist. Objektive „Fakten“ allein reichen nicht aus. Ohne eine subjektive Bewertung durch die jeweils betroffenen Einzelpersonen oder Gruppierungen liegt keine Wertschöpfung vor (ibid.). 
Entsprechend der von Meynhardt (2008; 2009) und Brämer et al. (2010) aufgezeigten Überlegungen zur Bedeutung individueller Sichtweisen, welche die abstrakte Vorgabe gesellschaftlicher Wertschöpfung konkret füllen, folgt die vorliegende Untersuchung einem Ansatz, wonach die Wahrnehmung einer gesellschaftlichen Wertschöpfung jeweils am individuellen Maßstab von vier Grundbedürfnissen erfolgt (Meynhardt 2009):

- instrumentell-utilitaristisch (Bedürfnis nach Orientierung und Handlungsspielraum, MittelZweck-Relationen)

- politisch-sozial (Bedürfnis nach positiven sozialen Beziehungen, Gruppenbeziehungen)

- moralisch-ethisch (Bedürfnis nach Selbstwerterhalt, Gleichbehandlung)

- hedonistisch-ästhetisch (Bedürfnis nach positiver Erfahrung, Wohlbefinden).

Den Grundgedanken, welcher psychologische Sichtweisen auf individuelle Bedürfnisse mit der Idee eines Public Value verknüpft, möchten wir zunächst an einem einfachen Beispiel verdeutlichen. Eine Organisation - so die Annahme - wirkt über ihr Kerngeschäft im gesellschaftlichen Umfeld zunächst in allen vier Bereichen. Sie prägt dieses somit aktiv mit. Ein Autohersteller schafft mit seinen Produkten nicht nur einen unmittelbaren Nutzen im Sinne instrumentell-utilitaristischer Erwartungen seiner Kunden (Gebrauchswert des Autos). Er beeinflusst ebenfalls deren Statusempfinden und die Gruppenidentifikation (politisch-soziale Bewertungsdimension), wie auch Genuss und Wohlbefinden (hedonistisch-ästhetische Bewertungsdimension) und das Selbstwertempfinden des Einzelnen (moralisch-ethische Bewertungsdimension). All dies geht weit über den Kundennutzen hinaus; es berührt die Sphäre gesellschaftlicher Werte und Normen. Wir sprechen von gesellschaftlicher Wertschöpfung / Public Value. Legitim ist, wenn ein Autohersteller, den Kunden ein attraktives Angebot unterbreitet und damit gleichzeitig akzeptierte gesellschaftliche Werte schafft oder zumindest nicht zerstört.

In dieser ganzheitlichen Konzeption finden unterschiedlichste Bewertungskriterien einen Platz. Eine normative Beurteilung der Kriterien findet hierbei nicht statt. Wie Tse (2011) vermerkt, spielt der Gedanke des Shareholder Value Managements in der unternehmerischen Praxis eine hervorgehobene Rolle. Dieser Rolle ist in multiperspektivischen Überlegungen zu Managementzielen ebenfalls Rechnung zu tragen. Um die Anregung Tses (2011) aufzunehmen und dem Gedanken einer strategischen Ausrichtung von Unternehmen entlang von Finanzkennzahlen (,Aktienwert“) gerecht zu werden, beziehen wir finanziell-ökonomische Erwägungen in der vorliegenden Untersuchung deshalb als zusätzliche Kategorie mit ein. Wir leiten diese Erwägungen als eigenständige Sub-Dimension aus der instrumentell-utilitaristischen Bewertungsdimension ab. Dies erscheint auch deshalb gerechtfertigt, da Autoren wie Edeling (2002) darauf verweisen, dass gerade in Bezug auf Überlegungen zum Gemeinwohl sachlich-instrumentelle Kategorien nicht mit fiskalischen bzw. finanziellen Kenngrößen verwechselt werden dürfen. Finanzieller und sachlicher Nutzen einer Unternehmung sind somit voneinander zu unterscheiden. Das gilt auch, wenn beide Kategorien aus psychologischer Perspektive ähnliche Funktionen erfüllen, indem sie Bedürfnisse nach Orientierung befriedigen und Auskunft über Mittel-Zweck-Relationen geben.

Der Public Value-Ansatz soll im Folgenden als Rahmen dienen, um die verschiedenen Bewertungskriterien in einen Zusammenhang zu stellen, welcher sich an menschlichen Grundbedürfnissen orientiert und auf diese Weise unternehmerisches Handeln aus Sicht der Gesellschaft beschreiben kann. 
Der Public Value im Spannungsfeld zwischen Aktienwert und Gemeinwohl.

\section{Hintergrund: Die Gruppe Deutsche Börse}

Die DBAG betreibt die FWB als Unternehmen innerhalb der GDB. Die GDB zeichnet sich durch ein integriertes Geschäftsmodell aus, das mit Dienstleistungsangeboten im Bereich Handel (Xetra, Eurex), Verrechnung (Eurex Clearing), Abwicklung und Verwahrung (Clearstream), entlang der ganzen Prozesskette des Wertpapierhandels vertreten ist (Deutsche Börse AG/Eurex Frankfurt AG 2012). Insgesamt arbeiten ca. 3.300 Mitarbeiter für die GDB in Deutschland, Luxemburg, der Schweiz und anderen europäischen Standorten, sowie in den USA und Asien. Der Hauptsitz der GDB liegt in Eschborn. Auf der von Forbes (2012) errechneten Liste der 2000 größten börsennotierten Unternehmen der Welt befand sich die Deutsche Börse im Jahr 2011 auf Rang 28 in Deutschland und Rang 714 im globalen Vergleich.

Aber nicht nur aufgrund ihrer Größe ist die GDB von Relevanz. Max Webers (1999 [1894]) Beobachtung in Bezug auf die ,ungeheure Bedeutung, welche die Börsen für die Volkswirtschaft... haben“ als deren „Regulatoren und Organisatoren“ (S. 160,) gilt heute mehr denn je (Weber/Davis/Lounsbury 2009; Davis 2009). Darüber hinaus fungieren Börsen als Symbol volkswirtschaftlichen Fortschritts und ökonomischer Prosperität (Morici 2011; Stoll 2006).

Das deutsche Börsensystem trägt der besonderen Rolle von Börsen Rechnung, indem es zwischen Börsenträgern und Börse unterscheidet. Börsen sind definiert als „Anstalten des öffentlichen Rechts mit eigenen Organen“ (Hessisches Ministerium für Wirtschaft, Verkehr und Landesentwicklung 2012). Als solche regeln sie ,ihre Strukturen, ihre Marktmodelle und die Nutzung ihrer Handelsplattformen durch Satzungen und Verwaltungsakte“ (ibid.). Die Tatsache, dass die GDB u. a. als Trägerin der FWB fungiert, und diese eine öffentlich-rechtliche Anstalt ist, impliziert, dass ,sie und ihre Organe hoheitlich [handeln] und... die Möglichkeit [haben], auch verwaltungsrechtliche Zwangsmittel anzuwenden“" (ibid.).

Gemäß Otto Mayers (1924) klassischer Definition des Handlungszwecks öffentlich-rechtlicher Organisationen besteht die Zielfunktion der FWB als Anstalt des öffentlichen Rechts demnach in der Realisierung öffentlicher Interessen. Aus unserer Sicht kann der wahrgenommene Beitrag dieser Realisierung als gesellschaftliche Wertschöpfung / Public Value verstanden werden.

Im Gegensatz zu seinen Regelungen der Rechtsform von Börsenorganisationen gibt das deutsche Börsengesetz keine explizite Rechtsform für Börsenträger vor. Somit konnte die 1990 gegründete Frankfurter Wertpapierbörse AG die Trägerschaft der FWB von der Industrie- und Handelskammer Frankfurt am Main im selben Jahr übernehmen. Nur zwei Jahre später ging aus der Frankfurter Wertpapierbörse AG die DBAG hervor, welche bis heute die FWB betreibt. Gehörte das Unternehmen zunächst dessen Kunden selbst, so ermöglichte der Börsengang der DBAG im Jahr 2001 eine sukzessive Ausweitung der Eigentümerstruktur, so dass sich die Aktien des Unternehmens mittlerweile vollständig im Streubesitz befinden. Dabei entfallen rund 95 Prozent der ausgegeben Aktien auf institutionelle Investoren, während die übrigen fünf Prozent von Privatanlegern gehalten werden.

Geographisch setzt sich die Eigentümerschaft der DBAG nur zu 18 Prozent aus deutschen Anteilseignern zusammen. Die übrigen Eigentümer stammen aus den USA (34 Prozent), Großbritannien (16 Prozent) und anderen Teilen der Welt (32 Prozent) (Deutsche Börse AG/Eurex Frankfurt AG 2012). 
Die Tatsache, dass vornehmlich internationale Finanzinvestoren und nicht etwa nationale und strategische Investoren - wie etwa im Fall der Beteiligung des Landes Niedersachsen an der Volkswagen AG - zu den Eigentümern der DBAG zählen, lässt vermuten, dass die Eigentümer des Unternehmens ihre Interessen weniger durch Gemeinwohlbeiträge des Unternehmens als vielmehr durch eine Steigerung von Kurswerten auf den Finanzmärkten gewahrt sehen. Diese Annahme teilt bereits Dodd Jr. (1932), der bemerkt: ,that stockholders who have no contact with business other than to derive dividends from it should become imbued with a professional spirit of public service is hardly thinkable“ (S. 1153). Öffentlich-rechtliche Vorgaben aus dem Börsenrecht bzw. ein öffentliches Interesse im Sinne der von Mayer (1924) formulierten Definition wirken somit ebenso auf die Manager im Unternehmen Deutsche Börse ein, wie die in Form von Finanzkennzahlen kommunizierten Eigentümererwartungen.

\section{Methodischer Ansatz}

\section{Auswahl der Fallstudie}

Unsere Untersuchung ist als Einzelfallstudie (vgl. Yin 2009) konzipiert. Die DBAG ist aufgrund ihrer institutionellen Strukturen und Entwicklung für unsere Studie besonders geeignet.

Die spezifische Konstellation, welche die DBAG als an der Börse notiertes Unternehmen und Betreiberin einer öffentlich-rechtlichen Anstalt kennzeichnet, legt allein aus formalen Gründen eine entsprechende Aufmerksamkeit innerhalb des Managements für diese Thematik nahe. Hinzu kommt auch ganz unabhängig von aktuellen Diskussionen über eine mögliche Börsenfusion eine erhöhte öffentliche Aufmerksamkeit für „die Börse“ als ein Symbol unserer Wirtschaftsordnung. Einer der beiden Forscher verfügt ferner über Organisationserfahrung, welche er im Rahmen mehrjährigen Mitarbeiterschaft als Werkstudent bei der DBAG sammeln konnte.

Von Seiten der DBAG bzw. GDB bestand zudem über Corporate Responsibility-Sichtweisen hinaus auch ein besonderes strategisches Interesse an der Fragestellung. Im Geschäftsbericht 2007 wurde dieses explizit formuliert: „Wir wollen Werte schaffen für Aktionäre, Kunden, Mitarbeiter und für die Gesellschaft insgesamt“ (Deutsche Börse AG 2008, keine Seitenzahl).

Diese Motivation des Forschungspartners, gesellschaftliche Fragestellungen als Bestandteil strategischer und operativer Fragestellungen zu erörtern, prägte die Zusammenarbeit im Forschungsprozess und ermöglichte uns guten Zugang zu den Interviewpartnern.

\section{Untersuchungsfeld}

In der Rekonstruktion des Public Values konzentrieren wir uns in dieser Studie auf die Akteursperspektive des mittleren Managements. Auf dieser Managementebene treffen unterschiedliche Spannungsfelder aufeinander und es müssen strategische und operative Überlegungen zusammengeführt werden (Woolridge/Schmid/Floyd 2008; Floyd/Woolridge 1997). Indem auf der Ebene des mittleren Managements unterschiedliche Wissensformen zusammentreffen, welche 
sich bei der Bewältigung von Herausforderungen im Managementalltag als handlungsleitend bewähren müssen, ist diese Ebene für die Theoriebildung eine besonders wichtige Erkenntnisquelle. Wir ordnen den Angehörigen dieser Ebene eine zentrale Funktion im Rahmen der Bewältigung mehrdimensionaler Ansprüche zu, mit denen Organisationen im benannten Spannungsfeld von Aktienwertsteigerungen und Gemeinwohlbeiträgen konfrontiert sind.

Aufgrund der Nähe zum operativen Geschäft können Manager der mittleren Führungsebene Veränderungen im Unternehmensumfeld in besonderer Weise wahrnehmen (vgl. Lawrence/Lorsch 1967, in: McMullen/Shepherd/Patzeld 2009). Sie sind dementsprechend befähigt, Interessenlagen einzelner Anspruchsgruppen zu identifizieren (Mintzberg 1994; Raman 2009). Ihnen kommt damit eine besondere Rolle bei internen Mediationsprozessen externer Ansprüche und deren Übersetzung in Handlungsrationalitäten der betroffenen Organisation zu (vgl. Woolridge/Schmid / Floyd 2008; Floyd/Woolridge 1997).

Insgesamt wurden 16 Manager der mittleren Führungsebene zwischen Mai 2009 und Juli 2010 im Hauptsitz der GDB befragt. Deren Auswahl fand in Absprache mit den Verantwortlichen des Strategiebereichs und der Corporate Social Responsibility-Abteilung statt. Ein Schwerpunkt wurde darauf gelegt, insbesondere Manager einzubeziehen, welche durch ihre Tätigkeit einen engen Kontakt zum gesellschaftlichen Umfeld haben (Tabelle A1 im Appendix).

$\mathrm{Zu}$ den Interviewpartnern gehörten somit die Verantwortlichen aller Bereiche mit unmittelbarer Berichtspflicht gegenüber dem Vorstandsvorsitzenden, die Hälfte der an den Finanzvorstand berichtenden Manager und jeweils ein Hauptverantwortlicher aus den Konzernfeldern „Xetra“, „Derivatives \& Market Data“, und „Clearstream“ sowie vier weitere leitende Angestellte aus den Bereichen „Human Resources“ (DBAG), „Operations/Infrastructure Management“ (Xetra) „Clearing/CCP Strategy“ (Eurex) und „Product Management/Design Core Products“ (Clearstream). Sieht man einmal vom Konzernfeld „Information Technology“ ab, waren somit Verantwortliche von mehr als einem Drittel aller unmittelbar an den Vorstand berichtenden Abteilungen an den Interviews beteiligt (Abbildung 1). 


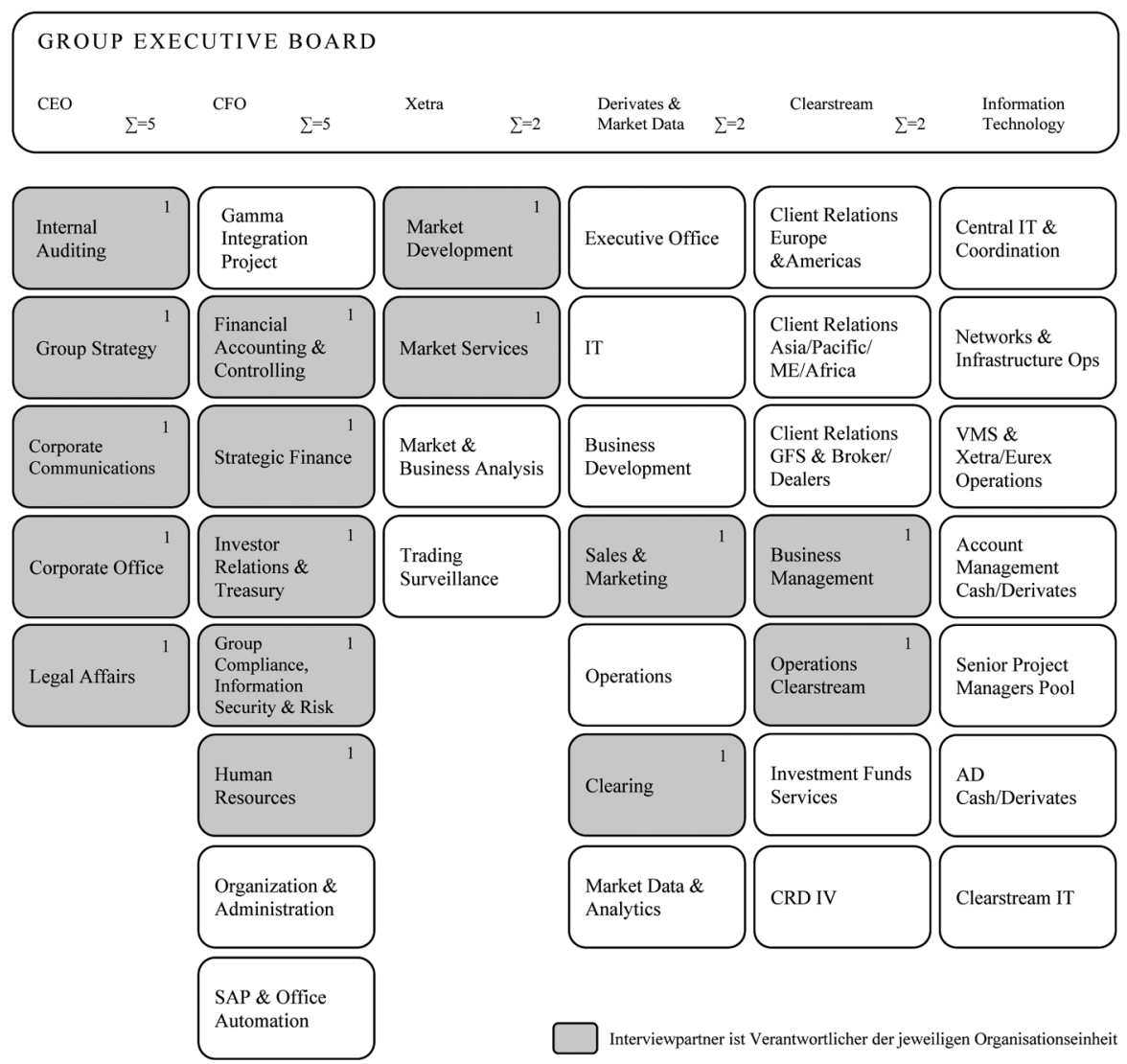

Abbildung 1: Befragte Verantwortliche mit unmittelbarer Berichtspflicht an den Vorstand nach Positionen innerhalb der GDB Konzernstruktur

Quelle: www.deutsche-boerse.com, eigene Hervorhebungen; Anzahl der befragten Manager in kursiven Zahlen, Summenzahlen je Spalte.

\section{Datenerhebung}

Unser Erkenntnisinteresse konzentrierte sich auf die Wahrnehmung von Wertbeiträgen durch Akteure innerhalb der GDB. Für die empirische Erhebung haben wir auf eine Variante des Repertory Grid-Verfahrens zurückgegriffen (vgl. Kelly 1955; Fransella/Bell/Bannister 2004). In der kognitiven Managementforschung werden diese Verfahren seit vielen Jahren erfolgreich eingesetzt (vgl. Walsh 1995; Huff 1997; Eden/Spender 1998). Insbesondere in explorativen Situationen erweisen sich diese Verfahren als geeignet, um neue Perspektiven und subjektiv relevante Bewertungsmuster strukturiert zu erheben.

Der von uns gewählte methodische Ansatz vereint Vorzüge idiographisch-qualitativer Herangehensweisen mit Möglichkeiten quantifizierender Methoden. Konkret wurde auf den von Meyn- 
hardt (2004) entwickelten „WertwissensGuide“ zurückgegriffen. Der WertwissensGuide basiert auf dem von Orlik (1979) entwickelten „Selbstkonzeptgitter“, welches den Grundgedanken von Kelly (vgl. 1955) aufnimmt und weiter entwickelt. Der WertwissensGuide trägt der subjektiven Realitätswahrnehmung der befragten Akteure dadurch Rechnung, dass die Befragten zunächst die aus ihrer Sicht relevanten Perspektiven in relativ freier Form einbringen können. Im Vergleich zum ursprünglichen Verfahren von Kelly (1955) erfolgt die Datenerhebung nach Orlik (1979) in vereinfachter Form. Die Bewertungsdimensionen werden vorab definiert - in unserem Fall in Form der fünf Nutzendimensionen. Die frei erhobenen Realitätsbeschreibungen (Konstrukte) werden in einem zweiten und letzten Schritt diesen Dimensionen zugeordnet.

Die Datenerhebung erfolgte in etwa 90-minütigen Interviews im Rahmen des beschriebenen Verfahrens. Die Antworten wurden durch die Forscher gemäß den methodischen Vorgaben aufgezeichnet.

Die von uns angewandte Vorgehensweise ist bereits in anderen Kontexten erprobt worden (Gomez/Meynhardt 2012; Meynhardt 2004; Schulze 2010). Wir möchten diese kurz skizzieren: Der Befragte wurde zuerst gebeten, die Grundfrage: „Was macht die GDB wertvoll für die Gesellschaft?“ aus verschiedenen Perspektiven zu beleuchten. Dazu sollte er jeweils drei positive (,So sollte es bleiben!“) und wünschenswerte Beiträge (,So sollte es werden!“) benennen. Diese waren von ihm zu ergänzen durch kritische (,So sollte es nicht bleiben!“) und ablehnungswürdige Aspekte (,So sollte es nicht werden!“).

Der Methode des WertwissensGuide folgend wurde der Befragte aufgefordert, jede seiner Aussagen durch Nennung des aus seiner Sicht wirksamen Gegenteils zu konkretisieren. Diese den in der Tradition von Kelly stehenden Interview-Verfahren eigene Vorgehensweise beruht auf der Annahme, dass die menschliche Wahrnehmung auf Gegensatzpaaren beruht (vgl. Kelly 1955). In Kenntnis des Gegenteils einer Aussage ist diese selbst besser verständlich: Was der Begriff „Kälte“ umschreibt, verdeutlicht sich erst und besser in Relation zum Gegenbegriff „Wärme“. Übertragen auf die Fragestellung gesellschaftlicher Wertbeiträge durch die Deutsche Börse beinhalten die von den befragten Managern der Börse benannten bipolaren Konstrukte, z. B. Fragen zur „Regulierung transparenter Märkte“ vs. „bilaterale Over the Counter-Strukturen“, zur strategischen Ausrichtung der Börse auf eine „Balance von Risiko-Minimierung und Umsatz-Maximierung“ vs. „einer einseitigen Verfolgung von Profitinteressen“ oder zur Funktion der „Börse als Liquiditäts-Hub mit Referenzfunktion“ vs. der Ausrichtung des Unternehmens als ,,wahllose Transaktionsmaschine".

Im zweiten Teil des Fragebogens sollten die Befragten Annahmen darüber treffen, welche der beiden Seiten jeder Aussage von der breiteren Öffentlichkeit eher wertgeschätzt würde. Dabei wurden die befragten Manager darum gebeten, in ihrer Bewertung entlang der oben genannten fünf Bewertungsdimensionen zu unterscheiden. Auch dazu ein Beispiel: Als ,zentrale Repräsentanz für den Finanzplatz in Deutschland“, wie ein Interviewpartner formulierte, leistet die DBAG sowohl moralisch-ethische, als auch politisch-soziale und hedonistisch-ästhetische Wertbeiträge. 


\section{Gütekriterien}

Qualitative Einzelfallstudien stehen immer vor der besonderen Herausforderung, ihren wissenschaftlichen Wert unter Beweis zu stellen (Gibbert/Ruigrok/Wicki 2008). Sie sind ebenso einer Reihe von Missverständnissen ausgesetzt, z. B. dem einer mangelnden Generalisierbarkeit (Flyvbjerg 2006). Für uns besteht ein wichtiges Kriterium darin, inwieweit der Beitrag eine ,generative capacity“ (Gergen 1976) besitzt und in der Lage ist, eine neue Perspektive zu eröffnen, die nicht nur nachvollziehbar ist, sondern auch weitergehendes Interesse am Gegenstand weckt.

Zur Erhöhung der Nachvollziehbarkeit und Plausibilität (vgl. Weick 1989) der einzelnen Fallstudienergebnisse haben wir unterschiedliche Maßnahmen ergriffen. So erhielten die Interviewpartner vor jedem Gespräch identische schriftliche Informationen zu Hintergrund und Zielen der Erhebung sowie den Interview-Fragebogen. Die Instruktion wurde jeweils am Beginn vorgelesen, um die Durchführungsobjektivität weiter zu erhöhen. Alle Interviews wurden auf Basis einer strukturierten Erhebungsmethodik (WertwissensGuide) durchgeführt. Die Erhebungsbögen wurden gemeinsam mit den Interviewpartnern ausgefüllt und diesen im Anschluss an die Gespräche zur Überprüfung und Bestätigung vorgelegt. Allein die von den Interviewpartnern überprüften und freigegebenen Fragebögen bilden die Grundlage für die vorliegende Untersuchung.

Beide Forscher haben die Kategorienbildung zur Verdichtung der individuellen Aussagen unabhängig voneinander vorgenommen. In den strittigen Fällen (ca. 10\%) wurde ein Konsens in der Diskussion erreicht. Im Rahmen einer kommunikativen Validierung (Flick 1996, S. 243; Arbnor/ Bjerke 1997, S. 234, vgl. auch die Erörterung der „,respondent validation“ bei Silverman 2006) wurden sowohl die durch Interviews entstandenen Beobachtungen als auch die abgeleiteten Ergebnisse ausgewählten Experten im Unternehmen studienbegleitend und im Anschluss an die Erhebung in einer Fokusgruppe vorgestellt. Ein wichtiger Indikator für die soziale Validität unserer Ergebnisse waren hierbei die positiven Reaktionen der Befragten. So bestätigte z. B. ein leitender Angestellter in einer internen Ergebnispräsentation den entwickelten Bezugsrahmen mit großer Zustimmung als eine treffende Beschreibung der relevanten strategischen Herausforderungen und hielt die Stimmigkeit mit dem internen Unternehmensleitbild ausdrücklich fest.

Inwieweit unsere Ergebnisse auf andere (Börsen-)organisationen übertragbar sind, ist in weiteren Studien zu untersuchen. Bedeutsamer ist für uns zunächst die Nachvollziehbarkeit der differenzierten Beschreibung und konzeptionellen Strukturierung eines Phänomens (Siggelkow 2007). Damit tritt die Frage nach der externen Validität in den Hintergrund. Stattdessen kommt dem künftigen Abgleich der Ergebnisse mit weiteren internen und externen Perspektiven (Triangulation) eine weitaus wichtigere Rolle zu.

\section{Datenanalyse}

Der WertwissensGuide bietet verschiedene Analysetechniken. In der vorliegenden Studie konzentrierten wir uns zunächst auf die Inhaltsanalyse (vgl. Mayring 2008) der Antworten auf die Frage: „Was macht in ihren Augen die GDB für die Gesellschaft wertvoll?“‘. Ergänzt um jeweilig 
Der Public Value im Spannungsfeld zwischen Aktienwert und Gemeinwohl.

genannte Handlungsalternativen lagen insgesamt 384 Aussagen (192 Konstruktpaare) vor. Diese individuellen Aussagen über die Wertbeiträge der GDB bilden die Analyseeinheit dieser Studie. Wir haben alle Konstruktpaare analysiert. Um unsere Ergebnisse zu ordnen, wurden zunächst allgemeine und börsenspezifische Wertbeiträge unterschieden. Diese Differenzierung spielt gerade in der Debatte um Konfigurationen im Finanzsektor und deren öffentlichen Legitimation eine maßgebliche Rolle (Ritholz 2013; Di Napoli/Bleiwas 2011; vgl. auch Friedman 2010). Die Kategorien wurden sowohl induktiv als auch deduktiv gebildet. Generell haben wir versucht, jedes Konstruktpaar jeweils einem in der Literatur bereits benanntem Thema zuzuordnen (deduktiv). In allen anderen Fällen wurde eine neue Kategorie gebildet (induktiv). Das Ergebnis der Analyse zeigt Tabelle 1 .

\begin{tabular}{lr}
\hline Thematische Referenzpunkte der analysierten Konstruktpaare & Anzahl Konstruktpaare (Total 192) \\
\hline Generische bzw. börsenunspezifische Themen, davon Nennungen zu: & 81 \\
- Innovation \& Wachstum & 22 \\
- Vermeidung einseitiger Stakeholderausrichtungen & 14 \\
- Internationalität der GDB & 10 \\
- Reputationsmanagement & 9 \\
- Themen des nachhaltigen Managements & 8 \\
- Aspekten des unternehmerischen Geschäftsmodells & 8 \\
- Standortfragen & 5 \\
Leistungen der GDB als Arbeitgeber & 5
\end{tabular}

\begin{tabular}{lr}
\hline Unternehmensspezifische bzw. börsenspezifische Themen, davon Nennungen zu: & 111 \\
- Markt- und Marktplatzfunktionen & 33 \\
- volkswirtschaftlichen und unternehmerischen Risiken & 20 \\
- Markttransparenz und Informationen & 20 \\
- Gestaltungs- und Regulierungsfunktionen als neutrale Partei & 14 \\
- einer Förderung der Aktienkultur in Deutschland & 1 \\
- einer Europäisierung von Abwicklungsdienstleistungen & 1 \\
- Konsolidierungstendenzen europäischer Wertpapiermärkte & 1 \\
- Lobbying- und Sales-Strategien & 1
\end{tabular}

\section{Tabelle 1: Inhaltliche Wertbeiträge der GDB}

In den jeweils genannten Wertbeiträgen können sich die befragten Manager auf mehr als nur eine der genannten Formen von Wertbeiträgen beziehen. Zwar haben wir die Wertbeiträge der jeweils relevanter erscheinenden Kategorie zugeordnet, die genannten Kategorien schließen einander jedoch nicht aus. Die Zahlen sind deshalb nicht als relative Gewichtung von Aufmerksamkeiten zu interpretieren.

In 81 der 192 der genannten Konstruktpaare beziehen sich die befragten Manager ausschließlich auf generische Wertbeiträge, die keinen spezifischen Bezug zu den Funktionen der GDB haben und auch von anderen Unternehmen und Organisationen in anderen Bereichen der Wirtschaft erbracht werden können.

In 111 der benannten Konstruktpaare benennen die befragten Manager zumindest einen Wertbeitrag der GDB, welcher sich mit spezifischen Börsenfunktionen in Verbindung bringen lässt. Dabei beziehen sich die Gesprächspartner immer wieder auch auf Aufträge des Gesetzgebers (z. B. Bundesministerium der Finanzen 2012). Insgesamt erwies sich eine Differenzierung zwischen ,generischen“ und ,unternehmensspezifischen“ Wertbeiträgen als hilfreich. Wir können 
festhalten, dass die befragten Manager die Zielorientierung der GDB nicht einseitig aufgrund eines generischen unternehmerischen Mehrwerts beschreiben.

Neben der inhaltsanalytischen Betrachtung haben wir die mehrdimensionale Zuordnung der Wertbeiträge innerhalb der Konstruktpaare analysiert. Ausgangspunkt hierbei war für uns die Frage, ob die befragten Manager zwischen unterschiedlichen Bewertungsdimensionen unterscheiden und wenn ja, wie sie dabei auftretende Konflikte auflösen. Unter Zuhilfenahme der oben eingeführten vier bzw. fünf Public Value-Dimensionen sollten die Befragten im zweiten Teil des Fragebogens somit eine mehrdimensionale Bewertung der gesellschaftlichen Legitimation der Gruppe Deutsche Börse vornehmen. An dieser Stelle sollte explizit deutlich werden, ob und welche Spannungsfelder in der inhaltlichen und strategischen Ausrichtung der GDB aus Sichtweise der befragten Manager existieren.

Tabelle 2 verdeutlicht anhand dreier konkreter Beispiele das Bewertungsverfahren. Zur Lesweise: „(X)“ bedeutet, dass der Befragte davon ausgeht, dass der genannte Wertbeitrag in dieser Dimension Nutzen stiftet. „,(O)“ bedeutet, dass die entsprechende Handlungsalternative als nützlich bewertet wurde. Der Befragte musste sich jeweils zwischen beiden Varianten entscheiden.

Enthält eine Zeile durchgängig identische Symbole ((X) oder (O)) interpretieren wir dies als Zeichen für Wertkongruenzen: die jeweils befragte Person geht davon aus, dass ein spezifischer Wertbeitrag in allen fünf Dimensionen der jeweils benannten Alternative vorzuziehen ist. Enthält eine Zeile unterschiedliche Symbole ((X) und $(\mathrm{O}))$, so bewerten wir dies als Ausdruck von Wertkonflikten: die befragte Person kommt in mindestens einer Dimension zu einer abweichenden Einschätzung, d. h. ein Wertbeitrag wird offensichtlich nicht durchgängig positiv oder negativ gesehen. Etwas kann zwar moralisch korrekt, aber politisch anstößig sein. Wir sprechen immer dann von einem Wertkonflikt, wenn für einen Wertbeitrag solche Diskrepanzen vorliegen. Nachfolgende Tabelle fasst die unterschiedlichen Möglichkeiten der Bewertung mit konkreten Beispielen aus der Studie zusammen. 
Der Public Value im Spannungsfeld zwischen Aktienwert und Gemeinwohl.

\begin{tabular}{|c|c|c|c|c|c|c|}
\hline \multirow[b]{2}{*}{$\begin{array}{c}\text { Schwerpunkt } \\
\text { (X) }\end{array}$} & \multirow[b]{2}{*}{$\begin{array}{l}\text { Handlungs- } \\
\text { alternative } \\
\text { (O) }\end{array}$} & \multicolumn{5}{|c|}{$\begin{array}{l}\text { Aus Sicht und im Meinungsbild der breiteren Öffentlichkeit: Schafft oder ermög- } \\
\text { licht eher der genannte Schwerpunkt (X) oder das Gegenteil (O)... }\end{array}$} \\
\hline & & $\begin{array}{c}\text {...sachlich- } \\
\text { inhaltlichen } \\
\text { Nutzen? }^{1}\end{array}$ & $\begin{array}{l}\text {...sozial- } \\
\text { zwischen- } \\
\text { menschli- } \\
\text { chen } \\
\text { Nutzen? }^{2}\end{array}$ & 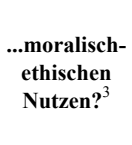 & $\begin{array}{l}\text {...angenehm- } \\
\text { positive } \\
\text { Erfahrun- } \\
\text { gen? }^{4}\end{array}$ & 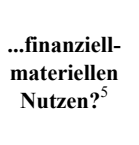 \\
\hline $\begin{array}{l}\text { 1. „Deutsche Börse } \\
\text { als Marktplatz Num- } \\
\text { mer I mit großer } \\
\text { volkswirtschaftlicher } \\
\text { Relevanz" }\end{array}$ & $\begin{array}{l}\text { „Beschränkung auf } \\
\text { Bedienung von } \\
\text { Teilmärkten" }\end{array}$ & $(\mathrm{X})$ & (X) & (X) & (X) & (X) \\
\hline $\begin{array}{l}\text { 2. „, Wahrnehmung } \\
\text { der Deutsche Börse } \\
\text { Group als Casino- } \\
\text { Betreiber" }\end{array}$ & $\begin{array}{l}\text { „Wahrnehmung } \\
\text { der GDB als fairer, } \\
\text { transparenter und } \\
\text { nutzenstiftender } \\
\text { Betreiber von } \\
\text { Marktinfrastruktu- } \\
\text { ren“ }\end{array}$ & $(\mathrm{O})$ & (O) & (O) & (O) & (O) \\
\hline $\begin{array}{l}\text { 3. „Zentrale Reprä- } \\
\text { sentanz für den Fi- } \\
\text { nanzplatz Deutsch- } \\
\text { land“ }\end{array}$ & $\begin{array}{l}\text { DBAG als norma- } \\
\text { les profitorientier- } \\
\text { tes Unternehmen } \\
\text { und ein Marktplatz } \\
\text { unter vielen" }\end{array}$ & (O) & (X) & (X) & $(\mathrm{X})$ & (O) \\
\hline
\end{tabular}

\section{Tabelle 2: Beispiele für Bewertungen von Konstruktpaaren}

${ }^{1}$ instrumentell-utilitaristische Dimension (Bedürfnis nach Orientierung, Mittel-Zweck-Relationen); ${ }^{2}$ politisch-soziale Dimension (Bedürfnis nach positiven sozialen Beziehungen, Gruppenbeziehungen); ${ }^{3}$ moralisch-ethische Dimension (Bedürfnis nach Selbstwerterhalt, Gleichbehandlung); ${ }^{4}$ hedonistisch-ästhetische Dimension (Bedürfnis nach positiver Erfahrung, Wohlbefinden); ${ }^{5}$ finanziell-ökonomische Subdimension der instrumentell-utilitaristischen Dimension.

Die Beispiele 1. und 2. in der Tabelle verdeutlichen das am häufigsten auftretende Bewertungsmuster. Innerhalb desselben bewerten Manager Wertbeiträge und Handlungsalternative einheitlich zustimmend oder ablehnend innerhalb der fünf Wert- bzw. Nutzendimensionen. Beispiel 3. zeigt einen Wertkonflikt bzw. ein Spannungsfeld auf: Während es aus sachlich-inhaltlicher und finanzieller Sicht für die Gesellschaft nützlich wäre, die Börse als „,normales profitorientiertes Unternehmen“" zu sehen, ist es in allen anderen Dimensionen offenkundig vorteilhafter, die Deutsche Börse als ,zentrale Repräsentanz für den Finanzplatz Deutschland“ zu betrachten.

Wir stellten fest, dass lediglich in 26 der insgesamt 192 bipolaren Konstrukte konfligierende Bewertungen vorliegen. Wir interpretieren diese Widersprüche in den Bewertungen der Befragten als Zeichen von Wertkonflikten in den jeweils konkreten Fällen. In der Mehrzahl der bewerteten Fälle (86,4 Prozent) gehen die Manager offenkundig davon aus, dass die Deutsche Börse in jeweils allen fünf Dimensionen gleichzeitig einen gesellschaftlichen Nutzen (Public Value) stiftet.

Nachfolgende Tabelle 3 listet alle Konstruktpaare auf, bei denen die befragten Manager Wertkonflikte im Rahmen der fünf abgefragten Dimensionen festgestellt haben. 
Timo Meynhardt und Camillo von Müller

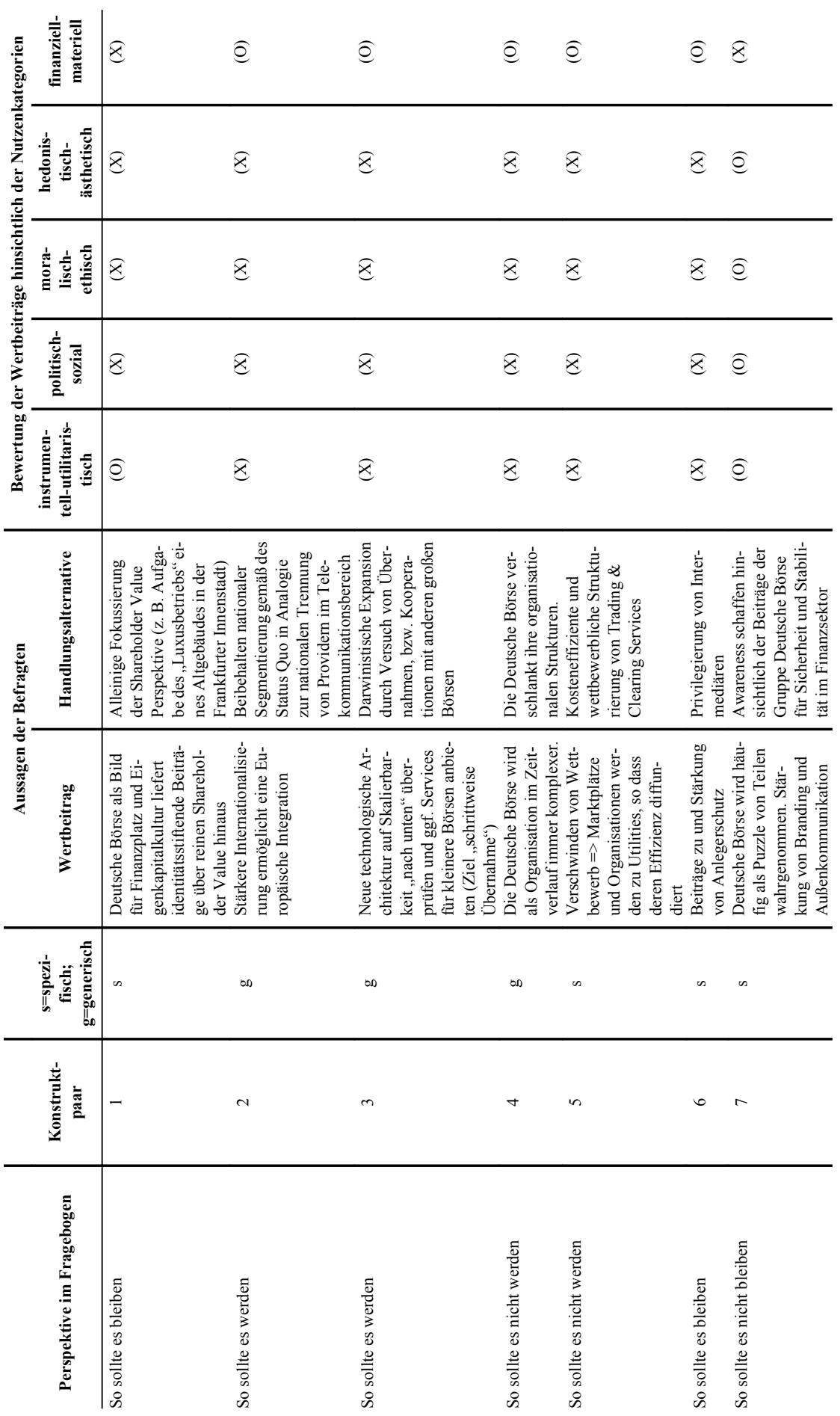


Der Public Value im Spannungsfeld zwischen Aktienwert und Gemeinwohl.

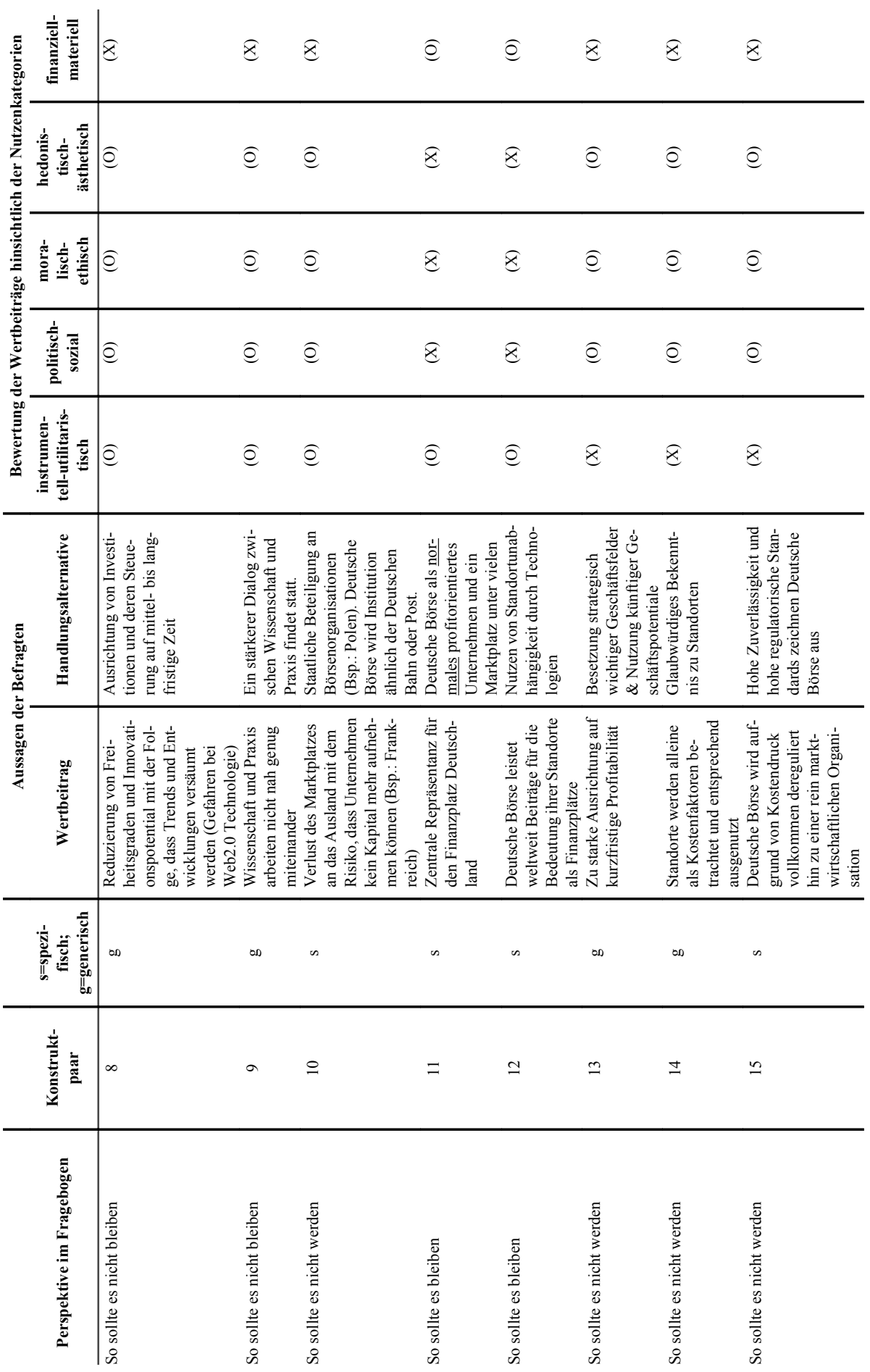


Timo Meynhardt und Camillo von Müller

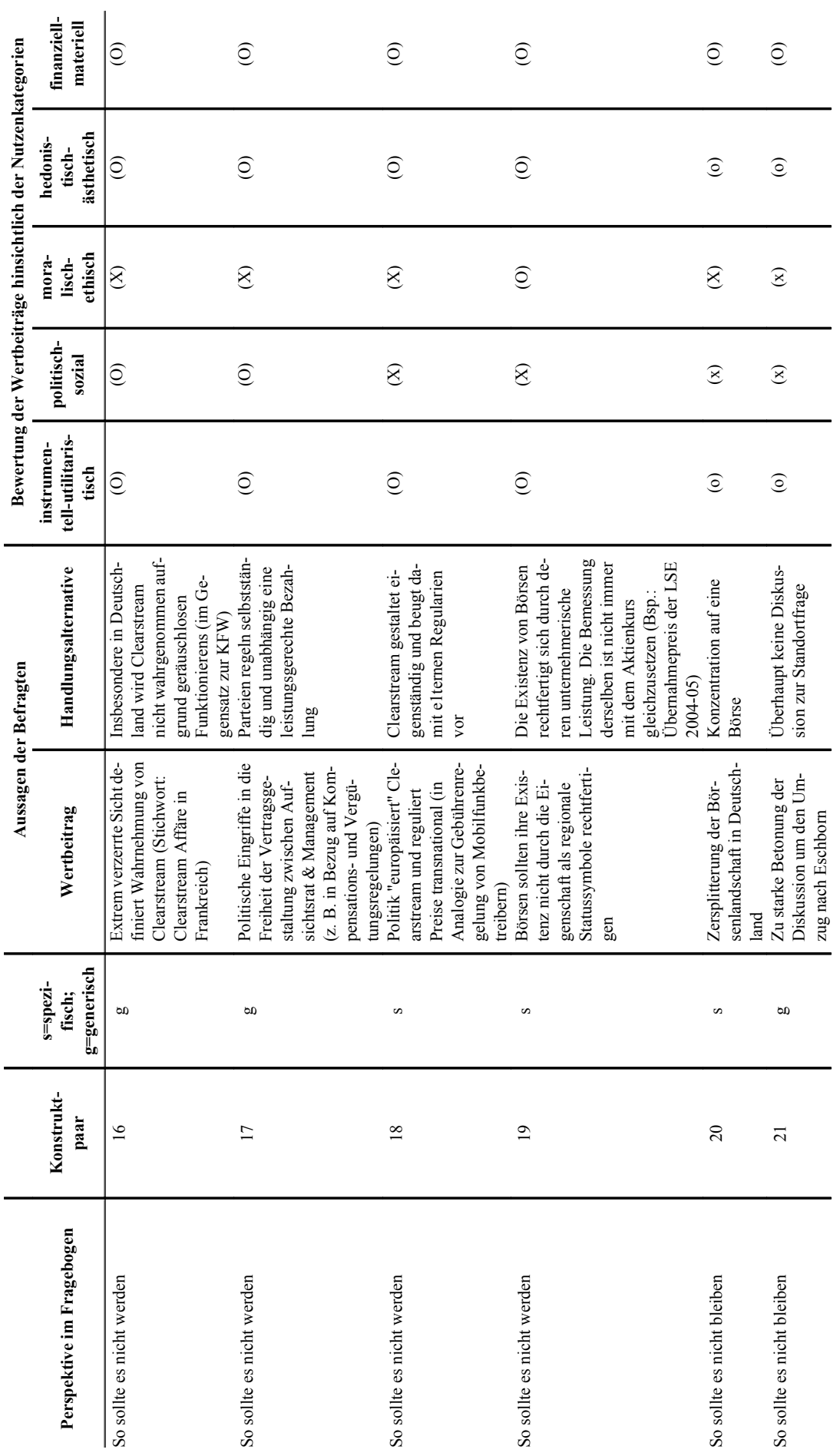


Der Public Value im Spannungsfeld zwischen Aktienwert und Gemeinwohl.

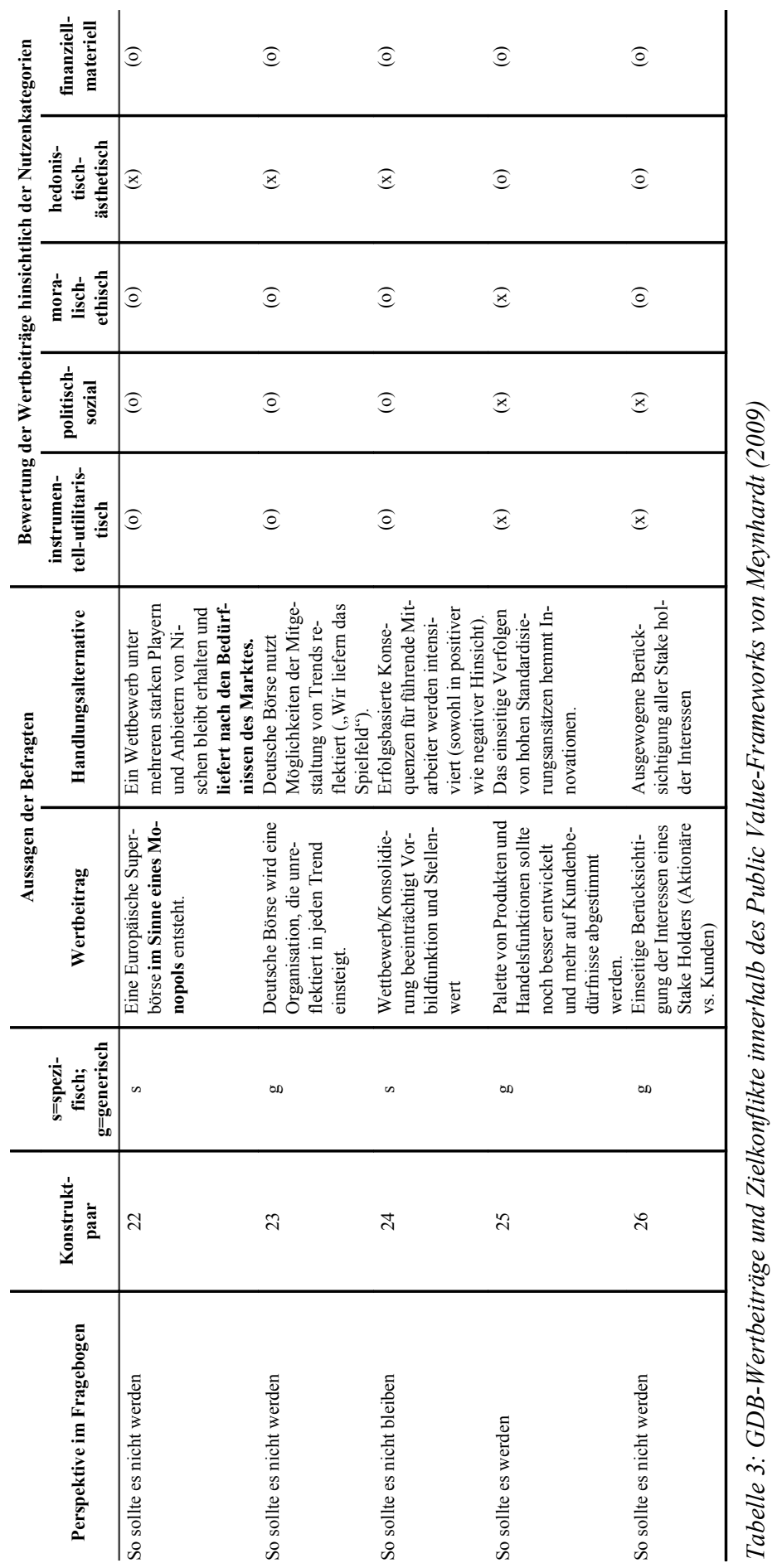


Die Tabelle ergibt folgendes Bild: Bei 15 der 26 Wertkonflikte geht es um Widersprüche zwischen finanziellen Nutzendimensionen, instrumentell-utilitaristischen Nutzendimensionen oder Kombinationen derselben einerseits und den übrigen drei bzw. vier Nutzendimensionen andererseits (Konstruktpaare 1-15). Ein Manager benannte eine „Ausrichtung auf kurzfristige Profitabilität“ als potentiellen Konfliktherd. Dabei war es aus Sicht des Befragten aus Sicht sachlicher und finanzieller Nutzenüberlegungen denkbar, diese strategische Zielvorgabe als legitimes Ziel einer auf Shareholder Value-Maximierung ausgerichteten Organisation zu benennen. Dem steht als Alternative eine „Besetzung strategisch wichtiger Geschäftsfelder und Nutzung künftiger Geschäftspotentiale" gegenüber, welcher aus Sicht politischer-sozialer und moralischer-ethischer Werte aber auch aus Sicht der hedonistisch-ästhetischen Werte der Vorzug zu geben wäre (Konstruktpaar 13).

Tabelle 3 zeigt weiterhin auf, dass die benannten 15 Konstruktpaare, in deren Kontext die befragten Manager Konfliktlinien entlang finanzieller bzw. instrumentell-utilitaristischer Dimensionen identifizieren, sowohl unternehmensspezifische Wertbeiträge (Konstruktpaare 1, 5, 6, 7, $10,11,12,15$ ) als auch generische Wertbeiträge (Konstruktpaare 2, 3, 4, 8, 9, 13, 14) enthalten. In sechs Fällen benannten befragte Manager Wertkonkurrenzen zwischen moralisch-ethischen Nutzendimensionen, politisch-sozialen Nutzendimensionen oder Kombinationen beider Kategorien einerseits und den übrigen Dimensionen andererseits (Konstruktpaare 16-21). Ein Beispiel für diese Art des Wertekonflikts: Ein Manager benannte die ,Zersplitterung der Börsenlandschaft in Deutschland“" als Situation, die aus politisch-sozialer und moralisch-ethischer Perspektive einer „Konzentration auf eine Börse“ vorzuziehen wäre. Letztere wäre gegenüber einer fragmentierten Börsenlandschaft jedoch sowohl aus instrumentell-utilitaristischer, finanziell-materieller als auch aus hedonistisch-ästhetischer Perspektive vorzuziehen (Konstruktpaar 20).

Die restlichen Konstruktbewertungen teilen sich auf wie folgt: In drei Fällen machen Interviewpartner Wertkonflikte zwischen der hedonistisch-ästhetischen Dimension und allen anderen aus (Konstruktpaare 22 - 24). Ein befragter Manager identifizierte in einem Fall einen Konflikt zwischen dem finanziell-materiellen und dem hedonistisch-ästhetischen Nutzen auf der einen und den übrigen Wertkategorien auf der anderen Seite (Konstruktpaar 25). Ein weiterer Interviewpartner identifizierte Spannungen zwischen politisch-sozialen und instrumentell-utilitaristischen Sichtweisen einerseits und den übrigen Wertdimensionen andererseits (Konstruktpaar 26).

Insgesamt ergibt sich aufgrund der nur geringen Anzahl von identifizierten Konfliktlinien zwischen den unterschiedlichen Wertdimensionen das Bild einer Organisation, deren mittlere Führungsebene mehr im Auge hat als das Ziel einer Steigerung des finanziellen Nutzens. In den Fällen, in denen jedoch Konfliktlinien zwischen unterschiedlichen Formen der Realisierung von Wertbeiträgen existieren, sind finanzielle Erwägungen eine der Hauptursachen für die von Managern wahrgenommenen Widersprüche zwischen unterschiedlichen Wertdimensionen.

\section{Ergebnisse}

Im Fokus unserer Untersuchung stand die Frage, wie Manager der GDB mit unterschiedlichen Ansprüchen umgehen, die sich aufgrund der besonderen Rolle als Finanzinstitution und Trägerin einer öffentlich-rechtlichen Anstalt für ihr Management ergeben. In einem ersten Schritt haben 
wir daher Wertbeiträge der GDB identifiziert, welche aus Sicht der befragten Manager das Unternehmen wertvoll machen für die Gesellschaft. In einem zweiten Schritt haben wir versucht zu erfahren, wie sich diese Beiträge im Rahmen eines mehrdimensionalen Bewertungsschemas zueinander verhalten. Dabei ging es auch darum nachzuvollziehen, entlang welcher Dimensionen des genannten Schemas aus Sicht der Befragten Wertkonflikte bestehen. Wie die Antworten im ersten Teil des Fragebogens zeigen, beschreiben die meisten der befragten Manager Wertbeiträge der GDB jenseits eindimensionaler Verweise auf die finanziellen Interessen ihres Unternehmens. Auch generische Wertbeiträge, wie etwa das von der GDB erwirtschaftete und geschaffene Steueraufkommen und Arbeitsplatzangebot, dominieren ihre Realitätskonstruktionen nicht. Stattdessen rechtfertigen die befragten Manager ihre Organisation vornehmlich durch die Identifikation konkreter börsenspezifischer Wertbeiträge.

Bei den Bewertungen im zweiten Teil des Fragebogens wird ein mehrdimensionaler Public Value sichtbar. Wie können die beschriebenen Wertbeiträge entlang der wichtigsten Spannungsfelder systematisiert werden? Zur Beantwortung dieser Frage haben wir die Antwortmuster in einem einheitlichen Bezugsrahmen geordnet. Dieser kombiniert inhaltliche Betrachtungen aus Teil 1 des Fragebogens zu unterschiedlichen Wertbeiträgen mit den unterschiedlichen Abwägungen aus Teil 2.

Eine erste Dimension ergibt sich aus der Bipolarität von generischen und unternehmensspezifischen Wertbeiträgen. In der zweiten Dimension möchten wir die Konflikte zwischen einzelnen Public Value-Dimensionen darstellen. Es sei zunächst daran erinnert, dass die Mehrheit aller Beiträge (86,4 Prozent) als durchgängig positiver Public Value gesehen wurde. Lediglich in 26 von 192 Fällen wurden Konflikte, insbesondere zwischen der finanziell-materiellen und der instrumentell-utilitaristischen Dimension einerseits und allen anderen Dimension andererseits deutlich. Auf der Suche nach handlungsleitenden Spannungsfeldern sehen wir darin eine Hauptkonfliktlinie, welche wir aufgreifen möchten. In der zweiten Dimension unterscheiden wir deshalb zwischen verschiedenen Gemeinwohlbeiträgen: Einen Pol bilden instrumentelle und finanzielle Beiträge, welche allerdings Gefahr laufen, dass sie nur Wenigen zugutekommen. Wir bezeichnen diese Seite als „Aktienwert“ - eine wichtige Dimension des Public Value, die aufgrund von Verteilungsproblemen jedoch Konfliktpotenzial in sich bergen kann. Den Gegenpol dazu bilden alle anderen Public Value-Dimensionen (moralisch-ethische, politisch-soziale und hedonistisch-ästhetische Beiträge), die offenkundig einer breiteren Öffentlichkeit zugutekommen. Wir bezeichnen diese Seite als „Gemeinwohl“ und meinen damit einen unstrittigen Public Value.

Kombinieren wir beide Dimensionen, so lassen sich vier Quadranten unterscheiden (A) - (D). Diese beschreiben unterschiedlich gewichtete Zielfunktionen und bilden den Bezugsrahmen zur Identifikation einzelner Spannungsfelder (Abbildung 2). 


\begin{tabular}{|c|c|}
\hline (A) & $\begin{array}{l}\text { sche } \\
\text { träge }\end{array}$ \\
\hline $\begin{array}{c}\text { Generische Steigerung des Aktienwertes (z.B. } \\
\text { Effizienzen in der Standortpolitik, schlanke } \\
\text { Unternehmensstrukturen) }\end{array}$ & $\begin{array}{c}\text { Generische Gemeinwohlbeiträge (z.B. mittel- bis } \\
\text { langfristig wirksame Investitionen, Steuern, } \\
\text { Arbeitsplätze) }\end{array}$ \\
\hline $\begin{array}{l}\text { Aktienwert (strittiger } \\
\text { Public Value) }\end{array}$ & $\begin{array}{l}\text { Gemeinwohl } \\
\text { (unstrittiger Public } \\
\text { Value) }\end{array}$ \\
\hline $\begin{array}{l}\text { Unternehmensspezifische Steigerung des } \\
\text { Aktienwertes (z.B. effiziente produkt- und } \\
\text { kundenorientierte Leistungen) }\end{array}$ & $\begin{array}{c}\text { Unternehmensspezifische Gemeinwohlbeiträge } \\
\text { (z.B. Vorbildfunktionen, } \\
\text { Stabilisierung marktlicher Strukturen) }\end{array}$ \\
\hline Unternehmens & sche Wertbeiträge \\
\hline
\end{tabular}

Abbildung 2: Bezugsrahmen der Wertbeiträge

Quelle: Eigene Abbildung.

Aus Sicht der befragten Manager ergänzen sich die meisten Wertbeiträge der GDB zu einem einheitlichen Public Value, der die Pole von Aktienwert und Gemeinwohl miteinander verbindet. Mit Hilfe des Bezugsrahmens können wir jedoch auch all jene Wertbeiträge beschreiben, welche nach Aussagen der befragten Manager Spannungen zwischen unterschiedlichen Unternehmenszielen bzw. an das Unternehmen herangetragenen Erwartungen aufzeigen. Dies sind Unternehmensbeiträge in Form...

a) ... eines generischen unternehmerischen Mehrwerts als Teil eines umfassenderen Public Value, der vornehmlich Eigentümern und internen Stakeholdern der GDB zugutekommt und deshalb strittig sein kann bzw. auf Kosten anderer Dimensionen geht. Beispiele sind eine alleinige Fokussierung auf kurzfristige Profitabilität (Konstruktpaar 13 in Tabelle 3) oder eine Sicht auf Unternehmensstandorte als bloße Kostenfaktoren (Konstruktpaar 14 in Tabelle 3);

b) ... eines generischen Public Value, der dem weiteren gesellschaftlichen Umfeld zugutekommt. In diesen Bereich gehören Themen, wie etwa Fragen der Internationalisierung (Konstruktpaar 2 in Tabelle 3), die Expansionsstrategien (Konstruktpaar 3), die interne Komplexität der GDB (Konstruktpaar 4 in Tabelle 3), die Ausrichtung von Investitionen und Steuerungsvorgaben entlang unterschiedlicher Zeithorizonte (Konstruktpaare 8, 13 in Tabelle 3), Vertiefungen des Dialogs zwischen Wissenschaft und Praxis (Konstruktpaar 9) sowie Fragen der Standortpolitik (Konstruktpaar 14 in Tabelle 3);

c) ... eines unternehmens- bzw. börsenspezifischen Public Value, den die GDB aufgrund ihrer spezifischen Konstellation als Finanzdienstleisterin und Börsenorganisation erbringt. Dazu gehören etwa Fragen der Referenz- und Vorbildfunktionen der Deutschen Börse (Konstruktpaare 1 und 11 in Tabelle 3), Fragen der Organisationsform der Deutschen Börse im Spannungsfeld von nicht-marktwirtschaftlich betriebenen „Utilities“ und deren marktwirtschaftli- 
che Organisation (z. B. Konstruktpaar 5 in Tabelle 3), das Informationsangebot der GDB zu ihrer systemischen und volkswirtschaftlichen Rolle (Konstruktpaar 7 in Tabelle 3), sowie Fragen der Nationalität der von der GDB betriebenen Standorte (Konstruktpaare 10 und 12 in Tabelle 3);

d) ... eines börsenspezifischen unternehmerischen Mehrwerts als Teil eines umfassenderen Public Value, der vornehmlich Eigentümern und internen Stakeholdern der GDB zugutekommt und deshalb strittig sein kann bzw. auf Kosten anderer Dimensionen geht. Diesen erbringt die GDB aufgrund ihrer spezifischen Konstellation als Finanzdienstleisterin und Börsenorganisation (z. B. die in Konstruktpaar 6 in Tabelle 3 angesprochene Option einer Privilegierung von Intermediären oder die in Konstruktpaar 15 in Tabelle 3 genannte Option einer vollkommenen Deregulierung der DBAG).

Je nach konkretem Sachzusammenhang schließen die Ziele in den einzelnen Quadranten einander aus oder ergänzen sich. So kann sich ein effizientes Angebot von Marktinfrastrukturen (Steigerung des Aktienwertes) positiv auf die zu zahlenden Steuern und die Schaffung von Arbeitsplätzen (Steigerung des Gemeinwohls) auswirken. Auch spezifische und generische Beiträge zum Aktienwert können einander ergänzen, wie die Vorgabe eines effizienten Angebots von Clearing \& Settlement Dienstleistungen deutlich macht.

Wie die Konstruktpaare 1-15 in Tabelle 3 aufzeigen, identifizieren die befragten Manager die Steigerung von Gemeinwohl und Aktienwert mitunter als widersprüchliche Zielvorgaben. Einander ausschließende Wertbeiträge sind etwa Leistungen der GDB, bzw. der DBAG, welche auf Profitmaximierung ausgerichtet sind, bei denen die DBAG aber aufgrund ihrer Rolle als Betreiberin einer öffentlich-rechtlichen Handelsplattform besondere Standards und Verpflichtungen beachten muss. So bezeichnet etwa ein Manager es als „Luxusbetrieb“, einen Börsensaal in einem Altgebäude der Frankfurter Innenstadt zu betreiben (Konstruktpaar 1 in Tabelle 3). Ein anderer Manager benennt Neutralitätsverpflichtungen der DBAG gegenüber Intermediären im Zuge des Anlegerschutzes, welcher der Profitmaximierung Grenzen setzt (vgl. Konstruktpaar 6 in Tabelle 3).

Die überwiegende Mehrzahl der 192 Konstruktpaare, welche die empirische Grundlage unserer Erörterungen darstellen, lassen keine Zielkonflikte erkennen. Eher wird darin ein produktives Zusammenspiel sichtbar. Ein Beispiel für sich ergänzende unternehmerische und gesellschaftliche Wertbeiträge sind von der DBAG innerhalb der GDB bereitgestellte Marktinfrastrukturen, die diese unter Beachtung von Synergie- und Skaleneffekten als profitorientierte Unternehmung betreibt. Daraus ergeben sich sowohl betriebswirtschaftliche Einsparungen (einzuordnen in Quadrant [D] in Abbildung 2), die als Effizienzen auch allgemein den Kapitalmärkten zugutekommen können (einzuordnen in Quadrant [C] in Abbildung 2; vgl. Aggarwal/Dahiya 2006; Francioni u. a. 2008). Dies impliziert jedoch nicht, dass es aufgrund dieser ,win-win-Situation“ beliebig wäre, ob die DBAG internen Verpflichtungen als Unternehmen oder externen Zielvorgaben als „Utility“ folgt. Vielmehr ist es gerade der Abwägungsprozess zwischen interner und externer Zielorientierung, der zu besagter Situation beiträgt.

Die genannten Beispiele machen deutlich, wie der aus den Daten abgeleitete Bezugsrahmen hilft, die unterschiedlichen Zielfunktionen innerhalb eines umfassenden Ordnungsrasters zu strukturieren. Strategische Zielvorgaben und Abwägungsnotwendigkeiten lassen sich somit in einem 
einheitlichen Rahmen gegenüber stellen, der sowohl unternehmerische als auch gesellschaftliche Aspekte berücksichtigt.

\section{Schlussfolgerungen}

Ausgangspunkt unserer Untersuchung war die Frage nach der gesellschaftlichen Wertschöpfung (Public Value) von Finanzorganisationen nach der Finanzkrise am Beispiel einer Börsenorganisation. Uns interessierte dabei, wie der Public Value aus Sicht des Managements konstruiert wird und inwieweit leitende Angestellte der GDB mögliche Widersprüche und Spannungsfelder wahrnehmen, die zwischen dem Ziel der Aktienwertsteigerung und formalen wie informellen Vorgaben zu Steigerungen des Gemeinwohls/Public Value bestehen können. Aus den unterschiedlichen institutionellen Verpflichtungen der GDB -,Aktienwert und Gemeinwohl “ - ergibt sich zunächst ein möglicher Zielkonflikt. Entscheidend für uns war jedoch nicht die formale Abgrenzung zwischen privatwirtschaftlicher Ausrichtung und öffentlichem Auftrag in den Statuten des untersuchten Unternehmens, sondern der wahrgenommene Public Value-Beitrag.

Die Daten legen nahe, dass die Befragten mit der Erfüllung der spezifischen Kernfunktionen einer Börse einen grundsätzlichen gesellschaftlichen Nutzen verbinden. Nur eine Minderheit verwies auf generische Funktionen der GDB als Arbeitgeberin oder Steuerzahlerin. Hingegen identifizierte die Mehrzahl der befragten Manager Wertbeiträge, welche spezifisch für die GDB sind etwa das Stabilisieren von Märkten, Schaffen von Transparenz und Senken von Allokationskosten.

Diese Realitätskonstruktionen interpretieren wir als Ausdruck für ein Wertebewusstsein, in dessen Rahmen die befragten Manager das Kerngeschäft der Börse als zutiefst gesellschaftsstabilisierende und -tragende Funktion innerhalb der sozialen Marktwirtschaft ansehen. Der Public Value ergibt sich demnach nicht so sehr durch einen allgemeinen Beitrag zum Wohlstand (Steuern und Abgaben), durch die Schaffung von Arbeitsplätzen oder gar durch zusätzliche Corporate Social Responsibility-Aktivitäten (Sponsoring, ehrenamtliche Tätigkeiten, etc.). Das mittlere Management sieht den eigentlichen Wertbeitrag vielmehr in der funktionalen Aufgabenerfüllung selbst, wie es bereits Max Weber (vgl. 1999 [1894]) formuliert hatte.

Die Ergebnisse der Analyse aus dem zweiten Teil des Fragebogens interpretieren wir dahingehend, dass die Befragten in der Mehrzahl der Fälle annehmen, dass ihre Sicht in der breiteren Öffentlichkeit geteilt wird. Sowohl aus instrumentell-utilitaristischer, politisch-sozialer, moralisch-ethischer, aber auch hedonistisch-ästhetischer und finanziell-ökonomischer Perspektive unterstellen sie eine hohe Wertschätzung für die DBAG in der Öffentlichkeit. Eine gesellschaftliche „licence to operate“ liegt aus Sicht der befragten Manager vor.

\section{Theorieimplikationen}

Der Tatsache, dass die befragten Manager nicht allein finanzielle Wertbeiträge ihres Unternehmens als wertstiftend ansehen, kommt vor dem Hintergrund normativer Positionen innerhalb der Managementliteratur besondere Bedeutung zu. Kontrovers ist dies vor allem aus Sicht jener Au- 
toren, die den wirtschaftlichen Aspekt zum Primat erheben. So befinden sich sowohl in der klassischen Argumentation Adol von Berle (1931) als auch nach Milton Friedman (vgl. 1970) Manager privatwirtschaftlicher Organisationen stets dann außerhalb ihres Legitimationsrahmens, wenn sie andere Ziele als die Aktienwertsteigerung verfolgen.

Da die DBAG auch Betreiberin der FWB und der Eurex Deutschland ist, liegt jedoch eine besondere Situation vor. Im Fall der DBAG steht somit Friedmans (vgl. 1970) Postulat der „Shareholder Value-Maximierung" als alleinige Aufgabe von Managern in privaten Unternehmen auch nicht im Widerspruch zu entgegenstehenden Definitionen von Managementaufgaben. Nach Davis (vgl. 2005) wiederum ergibt sich der Unternehmenszweck (,ultimate purpose of business“) aus der Bereitstellung von Produkten und Dienstleistungen, welche in der Gesellschaft nachgefragt werden (ibid. S. 112). Gemäß dieser Annahme sind es folglich sachlich-inhaltliche, nicht finanzielle Wertbeiträge, welche die Existenz von Unternehmen legitimieren.

Die DBAG sieht sich sowohl mit finanziellen Verpflichtungen gegenüber ihren Eigentümern als auch mit öffentlich-rechtlichen Ansprüchen konfrontiert. Diesem heterogenen Aufgabenrahmen entsprechend ergänzen im Fall der DBAG die Überlegungen Friedmans (vgl. 1970) jene von Davis (vgl. 2005) über gesellschaftspolitische Ansprüche an Unternehmen. Beide Perspektiven haben jedoch im Gegensatz zum Public Value-Ansatz den Nachteil, dass sie auf normativen Begründungsmustern aufbauen und deshalb nicht wertneutral sein können.

Auf Basis unserer Studie haben wir einen Bezugsrahmen vorgeschlagen, mit dessen Hilfe die Wertbeiträge der GDB strukturiert werden können. Dieser fasst unterschiedliche Wertbeitragsformen der DBAG innerhalb der GDB, bzw. der GDB selbst, zusammen. Aufgrund seiner Konzeption entlang der Kategorien ,generische vs. unternehmensspezifische Wertbeiträge“ und „Shareholder Value vs. Public Value“ ist der Bezugsrahmen auch auf andere Situationen übertragbar. Wir schlagen vor, diesen als Ordnungsraster für Fragen nach organisationalen, prozessualen und manageriellen Verhaltensweisen vor dem Hintergrund heterogener Anforderungen aus Wirtschaft und Gesellschaft zu nutzen. Sein Vorzug könnte darin bestehen, Spannungsfelder organisationaler Zielvorgaben in einer Managementperspektive abzubilden, die auch situative Anforderungen berücksichtigen kann.

In diesem Zusammenhang sind wir davon überzeugt, dass neue Perspektiven notwendig sind, um die Rolle des Finanzsektors in der Gesellschaft besser zu verstehen. Dabei kann der von uns entwickelte Bezugsrahmen besonderen Nutzen entfalten und auch die Theorieentwicklung anregen.

Dies möchten wir kurz erläutern. In seinem Artikel ,The Rise and Fall of Finance and the End of the Society of Organizations" diagnostiziert Gerald Davis (2009) fundamentale Wandlungsprozesse post-industrieller Ökonomien am Beispiel der US-Amerikanischen Wirtschaft. Er fasst diese so zusammen: „Where previously large corporations had been a dominant force ... through their employment practices, expansion choices, and community connections ... finance has increasingly taken center stage." (2009, S. 27) Damit wird ein Bild des Finanzsektors skizziert, dessen Institutionen von systemischer Relevanz für Gesellschaften und deren Volkswirtschaften sein können. So fragt Benjamin Friedman (2010) aus Sicht der Gesellschaft: „Is our Financial System Serving Us Well?“ Die Frage nach der Balance von gesellschaftlichen und unternehmerischen Interessen wird damit in den Vordergrund gerückt. 
Der von uns entwickelte Bezugsrahmen bietet in diesem Kontext einen Ansatzpunkt für die Strukturierung von unterschiedlichen Wertbeiträgen einer Organisation. Er zeigt auf, dass generische Wertbeiträge neben unternehmensspezifischen stehen. Es steht zu vermuten, dass sich eine „license to operate“ gerade im Finanzsektor nicht (mehr) allein durch unternehmensunspezifische Wertbeiträge (z. B. Steuern und Abgaben, Schaffung/Erhalt von Arbeitsplätzen) legitimieren lässt. Dies bedeutet z. B.: die gesellschaftliche Legitimität der in der New Yorker Wall Street angesiedelten Finanzunternehmen ist somit nicht alleine aufgrund von Arbeitsplätzen gegeben, die im Zuge von Multiplikatoreneffekten der dort gezahlten Gehälter in Stadt und Staat New York entstehen (vgl. Ritholz 2013; Di Napoli/Bleiwas 2011). Vielmehr sind es auch und gerade die spezifischen Gemeinwohlbeiträge von Finanzunternehmen, welche deren Existenz aus gesellschaftlicher Perspektive begründen. Insbesondere jene Perspektiven in der Organisationstheorie, welche die Einbettung einer Institution in ihren sozialen Kontext betonen, dürften sich hier als erkenntnisleitend erweisen (z. B. DiMaggio/Powell 1983). Der vorgeschlagene Bezugsrahmen hilft im Kontext dieser Überlegung, Schwerpunktsetzungen bzw. Leerstellen in Legitimationsund Abwägungsprozessen aufzudecken. Er gestattet es, sowohl gesellschaftsbezogene als auch unmittelbar unternehmensbezogene Sichtweisen einzunehmen und bei diesen zwischen komplementären und konkurrierenden Zielvorgaben an Unternehmen (z. B. ÖPP) zu unterscheiden. Wie unsere Ergebnisse zeigen, kann den Vertretern der mittleren Führungsebene in diesem Prozess eine wichtige Rolle zukommen. Ihre Nähe zum operativen Geschäft bringt einen tiefen Einblick in die sachlich-funktionale Leistungserbringung ihres Unternehmens. Bisherige Studien betonen insbesondere die Rolle des mittleren Managements für das strategische Management von Organisationen (vgl. Woolridge/Schmid/Floyd 2008). Diese Funktion wird ergänzt um eine Expertenrolle in der Standortbestimmung von Unternehmen in multidimensionalen Spannungsfeldern wirtschaftlicher und gesellschaftlicher Zielvorgaben und Interessen.

Die Rolle von „middle managers“ sollte somit auch in künftigen Studien zum Zusammenhang von Innovation, Wachstum und gesellschaftlicher Wertschöpfung eine stärkere Rolle spielen. Konsequenzen ergeben sich zudem für die Rolle von „middle managers“ in gesellschaftsbezogenen Managementprozessen in Unternehmen. So wie in den letzten Jahren (Woolridge/Schmid/ Floyd 2008) dem mittleren Management eine wichtige Rolle bei der Entwicklung neuer Ideen im Rahmen der strategischen Positionierung eines Unternehmens zugesprochen wurde, sollte dies auch für den Bereich der gesellschaftlichen Verantwortung (CSR) stärker genutzt werden.

\section{Praxisimplikationen}

Die GDB ist - so die Selbsteinschätzung ihres mittleren Managements - nicht nur finanziell erfolgreich. Sie ist auch - so die Ergebnisse unserer Befragung - in besonderer Weise zur gesellschaftlichen Wertschöpfung verpflichtet. Als Organisation versteht es die GDB somit augenscheinlich, informelle Erwartungen wie formale Vorgaben ihres Umfelds in managerielles Denken und Handeln zu überführen.

Die Existenz einer über die einzelnen Unternehmensbestandteile hinausgehenden Identität der „Gruppe Deutsche Börse“ ermöglicht es den Managern, Wertbeiträge integrativ zu reflektieren. Dies wird vor allen Dingen daran deutlich, dass die Befragten in ihren Sichtweisen auf das un- 
ternehmerische Umfeld nicht nur von den von ihnen selbst verantworteten Unternehmenseinheiten ausgingen. Vielmehr zielten sie darauf ab, die gesellschaftliche Position der GDB als Ganzes zu beurteilen. Unsere Untersuchungsergebnisse zeigen auf, welche Differenzierungen Manager in ihren Realitätskonstruktionen vornehmen. Es ist eine offene Frage, inwieweit Außenstehende diese nachvollziehen können und in gleicher Richtung bewerten.

In welchem Rahmen das komplexe Spannungsfeld zwischen Wirtschaft, Staat und Gesellschaft nicht nur eine Herausforderung für die DBAG ist, sondern eine solche Einbettung sogar ein Vorteil in der Kommunikation sein kann, bleibt abzuwarten. Der Dialog über die identifizierten Wertbeiträge gegenüber dem gesellschaftlichen Umfeld ist ganz sicher eine besondere Herausforderung für das Unternehmen. Dass dies als eine relevante Aufgabe erkannt wurde, zeigt sich etwa darin, dass die DBAG Anstrengungen unternimmt, ihre Funktion und Rolle gegenüber der Bundesregierung in Berlin oder auch auf europäischer Ebene in Brüssel darzustellen, wie die an beiden Orten von der Börse betriebenen Büros verdeutlichen.

\section{Limitationen und Ausblick}

Die vorliegende Einzelfallstudie erlaubt keine unmittelbare Generalisierung der Ergebnisse für andere Börsenorganisationen. Unsere Ergebnisse haben wir weder mit den Sichtweisen leitender Angestellten der obersten Führungsebene noch mit den Aussagen von Angehörigen anderer Managementstufen innerhalb des Unternehmens abgeglichen. Die Studie basiert allein auf der Selbstwahrnehmung einer systematisch ausgewählten Gruppe von Akteuren innerhalb der Organisation. In einer nächsten Forschungsphase sollte versucht werden, die gesellschaftliche Wertschöpfung auch aus externer Perspektive zu untersuchen. Künftige Untersuchungen müssen zeigen, inwieweit Diskrepanzen zu einer Fremdeinschätzung bestehen. Die Anwendung anderer Verfahren könnte dabei genutzt werden, um die Validität zu erhöhen bzw. Methodenartefakte zu erkennen. Ebenso gilt es weiter zu untersuchen, welche kognitiven Faktoren dazu beitragen, dass in der Selbstsicht relativ wenige Wertkonflikte wahrgenommen werden (Gomez/Meynhardt 2012). Erst ein Vergleich mit anderen Perspektiven kann offenlegen, welche Besonderheiten das Wertebewusstsein der befragten Manager aufweist. In der Public Value-Forschung finden sich erste Studien (z. B. Meynhardt/Bartholomes 2011), in denen Stakeholdergruppen zu konkreten Organisationen befragt werden. Es ist anzunehmen, dass ein solches Feedback für eine Organisation besonders dann relevant wird, wenn sie ihren Platz in der Gesellschaft bestimmen möchte.

Für die Theorieentwicklung sehen wir eine Herausforderung darin, das Zusammenspiel von generischen und unternehmensspezifischen Wertbeiträgen besser zu verstehen und zu erklären. Für die gesellschaftliche Akzeptanz und Legitimation von Institutionen im Finanzsektor erachten wir dies als eine zentrale Frage. 
Abstract

Timo Meynhardt and Camillo von Müller; „,We Want to Create Value for Society” - The Public Value between Stock Value and Common Good. The Case of Deutsche Börse AG

\section{Common Good; Management; Public Value; Stock Exchange}

Deutsche Börse AG is more than ,just “ a for-profit company. It also operates two public entities in form of Frankfurter Wertpapierbörse and Eurex Deutschland. The dual character of Deutsche Börse implies that its managers have to justify their operations both in financial as well as in societal terms. What are elements of the value-added that Deutsche Börse produces for society (Public Value) according to its managers? What is the relationship among these elements? The present paper discusses these questions based on a qualitative case study. The results show that Deutsche Börse managers primarily legitimize Deutsche Börse AG in societal terms by referring to its functional qualities as exchange. Against the background of empirical evidence, the resulting framework allows us to differentiate between competing and complementary elements of public value contributions. We discuss resulting implications both for theory and practice.

\section{Literaturverzeichnis}

Aggarwal, Reena/Dahiya, Sandeep (2006), Demutualization and Public Offerings of Financial Exchanges, in: Journal of Applied Corporate Finance, 18 (3), S. 96-106.

Arbnor, Ingeman/Bjerke, Björn (1997), Methodology for Creating Business Knowledge, 2nd Edition, London, New Dehli: SAGE.

Berle, Adolf. A. (1931), Corporate Powers as Powers in Trust, Harvard Law Review, XLIV (7), S. 1049-1074.

Brämer, Patrick/Gischer, Horst/Pfingsten, Andreas/Richter, Toni (2010), Der öffentlichen Auftrag der deutschen Sparkassen aus der Perspektive des Stakeholder Managements, in: Zeitschrift für öffentlichen und gemeinwirtschaftliche Unternehmen, 33. Jg. Heft 4, S. 311-332.

Bruch, Heike/Sattelberger, Thomas (2001 a), Lufthansa's Transformation Marathon. A Process of Liberating and Focusing Change Energy, in: Human Resource Management, 40 (3), S. 249-259.

Bruch, Heike/Sattelberger, Thomas (2001 b), Leading Change - Lufthansa's Way of Sustainable Reorientation, in: Journal of Change Management, 1 (4), S. 344-363.

Bundesministerium der Finanzen (2012), Geordnete Bahnen für die europäischen Finanzmärkte, www.bundesfinanzministerium.de, Stand: 27.3.2013.

Davis, Gerald (2009), The Rise and Fall of Finance and the End of the Society of Organizations, in: Academy of Management Perspectives, 23 (3), S. 27-44.

Davis, Ian(2005), What is the Business of Business?, in: The McKinsey Quarterly, 3, S. 105-113.

Davis, Lance / Neal, Larry (1998), Micro Rules and Macro Outcomes: The Impact of Micro Structure on the Efficiency of Securities Exchanges, London, New and Paris, in: The American Economic Review 88 (1998) 2, S. 40-45.

Deutsche Börse AG (2008), Just Value. Geschäftsbericht (2007), Frankfurt a.M: Deutsche Börse AG.

Deutsche Börse AG/Eurex Frankfurt AG (2012), Über uns. Online verfügbar unter: http://www.deutsche-boerse.com, Stand: 19.2.2012.

DiMaggio, Paul J./Powell, Walter W. (1983), The Iron Cage Revisited: Isomorphism and Collective Rationality in Organizational Fields. American Sociological Review. Vol 48, S. 147-160.

Di Napoli, Thomas P./Bleiwas, Kenneth B. (2011), The Securities Industry in New York City, Report 12-2012, Office of the New York City State Controller, October 2011.

Dodd Jr., E. Merrik (1932), For Whom Are Corporate Managers Trustees?, Harvard Law Review, XLV (7), S. 1145-1163.

Donaldson, Thomas/Dunfee, Thomas W. (1999), Ties That Bind. A Social Contract Approach to Business, Boston, MA: Harvard Business School Press.

Edeling, Thomas (2002). Stadtwerke zwischen Markt und Politik, in: Zeitschrift für öffentliche und gemeinwirtschaftliche Unternehmen, 25. Jg. Heft 2, S. 127-139. 
Der Public Value im Spannungsfeld zwischen Aktienwert und Gemeinwohl.

Eden, Colin/Spender, J.C. (Hrsg. 1998), Managerial and Organizational Cognition. Theory, Methods, and Research, Thousand Oaks, CA, USA: Sage.

Eisenhardt, Kathlen M. \& Graebner, M. E. (2007), Theory building from cases: opportunities and challenges. Academy of Management Journal, 50(1): 25-32.

Fischer, Markus / Hainz, Christa / Rocholl, Jörg / Steffen, Sascha (2012), Government Guarantees and Bank Risk Taking Incentives. AFA 2012 Chicago Meetings Paper.

Flick, Udo (1996), Qualitative Forschung: Theorie, Methoden, Anwendung in Psychologie und Sozialwissenschaften. Reinbek: Rowohlt Taschenbuch.

Floyd, Steven/Woolridge, Bill (1997), Middle Management's Strategic Influence and Organizational Performance, in: Journal of Management Studies, 34 (3), S. 465-485.

Flyvbjerg, Bent (2006), Five Misunderstandings About Case-Study Research, Qualitative Inquiry, Volume 12, Nr. 2, April, S. 219-245.

Forbes (2012), The World's Biggest Public Companies. Online verfügbar unter: http://www.forbes.com/global2000/ list/, Stand: 19.2.2012.

Francioni, Reto/Hazarika, Sonali, Reck, Martin/Schwartz, Robert (2008), Equity Market Microstructure: Taking Stock of What We Know, in: Journal of Portfolio Management, 35 (1), S. $57-71$.

Fransella, Fay/Bell, Richard/ Bannister, Don (2004), A Manual for Repertory Grid Technique, Chichester, UK: John Wiley and Sons.

Freixas, Xavier (2010), Post-Crisis Challenges to Bank Regulation. Economic Policy, Vol. 25 (62), S 375-399.

Friedman, Benjamin M. (2010), Is Our Financial System Serving Us Well?, Daedalus, Journal of the American Academy of Arts and Sciences, Fall, S. 9-21.

Friedman, Milton (1970), The Social Responsibility of Business Is to Increase Its Profits, in: The New Times Magazine, September 13 .

Gergen, Kenneth (1978), Toward Generative Theory. in: Journal of Personality and Social Psychology, 36(11), 1344-1360.

Gibbert, Michael, Ruigrok, Winfried. \& Wicki, Barbara (2008), What Passes as a Rigorous Case Study? in: Strategic Management Journal, 29(13), S. 1465-1474.

Gibson, George Rutledge, 1891: Wall Steet. The Utilities and Ethics of Speculation, the Stock Exchange as an Economic Factor, Address Before the Convention of American Bankers at New Orleans, on Thursday, November 12, Atlanta: Lookwood Press, 1891.

Gomez, Peter/Meynhardt, Timo (2012), More Foxes in the Boardroom: Systems Thinking in Action, In: Stefan Groesser/Rene Zeier, Systemic Management for Intelligent Organizations: Concepts, Model-Based Approaches, and Applications, Heidelberg, New York : Springer Verlag, S. 83-98.

Hessisches Ministerium für Wirtschaft, Verkehr und Landesentwicklung (2012), Börsenaufsicht. Online verfügbar unter: http://www.wirtschaft.hessen.de, Stand: 19.2.2012, Stand: 19.2.2012.

Huff, Anne S. (1997), A Current and Future Agenda for Cognitive Research in Organizations, in: Journal of Management Studies, 34, S. 947-952.

Kelly, George A. (1955), The Psychology of Personal Constructs: Vol. 1, London: Norton.

Knight, Frank H. (1982 [1939]), Social Science and the Political Trend, in: Knight, Frank H., 1982: Freedom and Reform. Essays in Economics and Social Philosophy, Indianapolis: Liberty Fund.

Lawrence, Paul R./Lorsch, Jay, W. (1967), Differentiation and Integration in Complex Organizations, in: Administrative Science Quarterly 12, S. 1-30.

Mayer, Otto (1924), Deutsches Verwaltungsrecht, 2. Auflage, Berlin: Duncker und Humblot.

Mayring, Philipp (2008), Qualitative Inhaltsanalyse: Grundlagen und Techniken, 10. Auflage, Weinheim: Beltz.

McMullen, Jeffrey S./Shepherd, Dean A./Patzelt, Holger (2009), Managerial (In)Attention to Competitive Threats, in: Journal of Management Studies, 46 (2), S. 157-181.

Meynhardt, Timo (2004), Wertwissen: Was Organisationen wirklich bewegt, Münster: Waxmann.

Meynhardt, Timo (2008), Public Value: Oder was heißt Wertschöpfung zum Gemeinwohl?, in: der moderne Staat, 2(2), S. 73-91.

Meynhardt, Timo (2009), Public Value Inside: What is Public Value Creation?, in: Journal of Public Administration, 32 (3), S. 192-219.

Meynhardt, Timo/Bartholomes, Steffen (2011), (De)Composing Public Value: In Search of Basic Dimensions and Common Ground, in: International Public Management Journal, 14:3, S. 284-308.

Meynhardt, Timo/Metelmann, Jörg (2009), Pushing the Envelope: Creating Public Value in the Labor Market: An Empirical Study on the Role of Middle Managers, in: International Journal of Public Administration, 32, S. 274-312.

Mintzberg, Henry (1994), The Rise and Fall of Strategic Planning. Reconceiving Roles for Planning, Plans, and Planner, New York: The Free Press.

Morici, Peter (2011) German Takeover of NYSE Would Be Bad for U.S., in: FOXBusiness. Online verfügbar: http:// www.foxbusiness.com, Stand: 19.2.2012.

Orlik, Peter (1979), Das Selbstkonzept als Bezugssystem sozialer Kognitionen., in: Zeitschrift für Sozialpsychologie, 10, S. 167-182. 
O'Sullivan, Niam/ O'Dwyer, Brendan (2009), Stakeholder Perspectives on a Financial Sector Legitimation Process: The Case of NGOs and the Equator Principles, in: Accounting, Auditing \& Accountability Journal, Vol. 22 (4), S. 553-587.

Pirson, Michael / Turnbull, Shann (2011), Corporate Governance, Risk Management, and the Financial Crisis: An Information Processing View, in: Corporate Governance: An International Review, 2011, 19(5), S. 459-470.

Preda, Alex (2009), Framing Finance. The Boundaries of Markets and Modern Capitalism, Chicago: University of Chicago Press.

Raman, S. Raghu (2009), Middle Managers' Involvement in Strategic Planning: An Examination of Roles and Influencing Factors, in: Journal of General Management, 34 (3), S. 57-74.

Ritholtz, Barry (2013), Defending Wall Street Bonuses, in: The Big Picture, ww.ritholtz.com/blog/2013/03/defending-wall-street-bonuses/, Stand: 27.3.2013.

Schanz, Kai-Uwe (2009), Maintaining Stakeholder Trust in Difficult Times. Some Fundamental Reflections in Light of the Credit Crisis, in: The Geneva Papers, 2009, 34, S. 260-270.

Schulze, Eric (2010), Der Beitrag zum Gemeinwohl: Public Values definieren und legitimieren. Eine Untersuchung am Beispiel des Goethe-Instituts e.V. Dissertation der Universität St. Gallen, Hochschule für Wirtschafts-, Rechts- und Sozialwissenschaften (HSG), Borsdorf: Edition Winterwork.

Schumpeter, Joseph A. (2008 [1934]), The Theory of Economic Development. An Inquiry into Profits, Capital, Credit, Interest, and the Business Cycle. Translated from the German by Redvers Opie with a New Introduction by John E. Elliot, New Brunswick, USA: London, UK: Transaction Publishers.

Siggelkow, Nicolaj (2007), Persuasion With Case Studies, Academy of Management Journal, 50(1), S. 20-24.

Silverman, David (2006), Interpreting Qualitative Data, Third Edition, London, New Delhi: Sage.

Sornette, Didier and Von der Becke, Susanne (2011), Crashes and High Frequency Trading, Swiss Finance Institute Research Paper No. 11-63.

Stoll, Hans R. (2006), Electronic Trading in Stock Markets, in: The Journal of Economic Perspectives, 20 (1), S. 153-174.

Summermatter, Lukas (2012), Die Schweizerischen Bundesbahnen als Allzweckinstrument der Politik, in: Zeitschrift für öffentliche und gemeinwirtschaftliche Unternehmen, 35. Jg. Heft 1, S. 76-92.

Tse, Terence (2011), Shareholder and Stakeholder Theory: After the Financial Crisis", Qualitative Research in Financial Markets, Vol. 3 (1), S. 51-63.

Walsh, James P. (1995), Managerial and Organizational Cognition: Notes from a Trip Down Memory Lane, in: Organization, Science, 6(3), S. 280-321.

Weber, Klaus/Davis, Gerald F./Lounsboury, Michael (2009), Policy as Myth and Ceremony? The Global Spread of Stock Exchanges 1980 - 2005, in: The Academy of Management Journal, 52 (6), S. 1319-1368.

Weber, Max (1999 [1894]), Die Börse, in: Weber, Max, 1999: Börsenwesen. Schriften und Reden 1893-1898. Herausgegeben von Knut Borchardt in Zusammenarbeit mit Cornelia Meyer-Stoll, Tübingen: J.C.B. Mohr [Paul Siebeck], S. 127-174.

Werhane, Patricia/Hartman, Laura/Archer, Crina/Bevan/David/Clark/Kim (2011), Trust after the Global Financial Meltdown, in: Business and Society Review 116 (4), S. 403-433.

Weick, Karl, (1989), Theory Construction as Disciplined Imagination. in: Academy of Management Review, 14(4), 516-531.

Weihe, Guŏrio (2008), Public-Private Partnerships and Public-Private Value Trade-Offs, in: Public Money and Management, 28 (3), S. 153-158.

Windisch, Rupert (Hrsg, 1987), Privatisierung natürlicher Monopole im Bereich von Bahn, Post und Telekommunikation, Tübingen: J.C.B. Mohr [Paul Siebeck].

Woolridge, Bill/Schmid, Torsten/Floyd, Steven (2008), The Middle Management Perspective on Strategy Process. Contributions, Synthesis, and Future Research, in: Journal of Management, 34, S. 1190-1221.

Yin, Robert K. (2009), Case Study Research. Design and Methods, Fourth Edition, Los Angeles u. a.: Sage. 
Der Public Value im Spannungsfeld zwischen Aktienwert und Gemeinwohl.

\section{APPENDIX}

$\begin{array}{ll}\begin{array}{l}\text { Entity / Divisi- } \\ \text { on }\end{array} & \text { Area / Department } \\ \text { DBAG / CEO } & \text { Group Strategy } \\ \text { DBAG / CEO } & \text { Internal Auditing } \\ \text { DBAG / CEO } & \text { Corporate Communications } \\ \text { DBAG / CEO } & \text { Group Corporate Office } \\ \text { DBAG / CEO } & \text { Legal Affairs } \\ \text { DBAG / CFO } & \text { Financial Accounting \& Controlling } \\ \text { DBAG / CFO } & \text { Strategic Finance } \\ \text { DBAG / CFO } & \text { Investor Relations \& Treasury } \\ \text { DBAG / CFO } & \text { Group Compliance, Information Security } \\ & \text { \& Risk Management } \\ \text { DBAG / CFO } & \text { Human Resources } \\ \text { DBAG / Xetra } & \text { Market Development } \\ \text { DBAG / Xetra } & \text { Operations / Infrastructure Development } \\ \text { Eurex / Deriva- } & \text { Sales \& Marketing } \\ \text { tives \& Markets } & \\ \text { Eurex / Deriva- } & \text { Clearing / CCP \& Strategy } \\ \text { tives \& Markets } & \\ \text { Clearstream } & \text { Product Management \& Design Core } \\ \text { Banking AG / } & \text { Products } \\ \text { Clearstream } & \\ \text { Clearstream } & \text { Operations Clearstream } \\ \text { Banking AG / } & \\ \text { Clearstream } & \end{array}$

\section{Funktion}

MD/Head of Area Director Area Group Strategy Head of Section Internal Auditing

Head of Department Corporate Communications Head of Department Group Corporate Office MD/Head of Area Director Legal Affairs

MD Head of Area Financial Accounting \& Controlling Head of Section Strategic Finance

Head of Department Investor Relations \& Treasury

Head of Department Group Compliance, Information Security \& Risk Management

Head of Section Human Resources Germany \& Corporate Training MD/Head of Area Director Market Development

Head of Department Operations / Infrastructure Development

Head of Department Sales \& Marketing

Head of Department Clearing / CCP \& Strategy

Head of Department Product Management \& Design Core Products

Head of Department Operations Clearstream

\section{Tabelle A1: Befragte Manager nach Positionen innerhalb der GDB}

